\title{
The EUI instrument on board the Solar Orbiter mission: from breadboard and prototypes to instrument model validation
}

\author{
J.-P. Halain ${ }^{a^{*}}$, P. Rochus ${ }^{\mathrm{a}}$, E. Renotte ${ }^{\mathrm{a}}$, T. Appourchaux ${ }^{b}$, D. Berghmans ${ }^{g}$, L. Harra $^{e}$, U. Schühle $^{d}, \mathrm{~W}$.

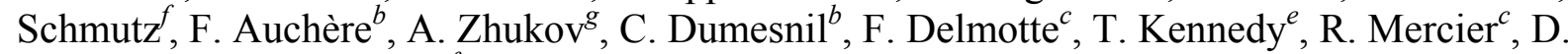

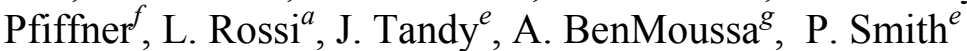 \\ ${ }^{a}$ Centre Spatial de Liège, Université de Liège, Liege Science Park, 4013 Angleur, Belgium \\ ${ }^{\mathrm{b}}$ Institut d'Astrophysique Spatiale, Orsay, France \\ 'Institut d'optique, Orsay, France \\ ${ }^{\mathrm{d}}$ Max-Planck-Institut für Sonnensystemforschung, Katlenburg-Lindau, Germany \\ ${ }^{\mathrm{e}}$ Mullard Space Science Laboratory, Surrey, UK \\ ${ }^{\mathrm{f}}$ Physikalisch-Meteorologisches Observatorium Davos and World Radiation Center, Switzerland \\ ${ }^{\mathrm{g}}$ Royal Observatory of Belgium, Avenue Circulaire, Uccle, Belgium
}

\begin{abstract}
The Solar Orbiter mission will explore the connection between the Sun and its heliosphere, taking advantage of an orbit approaching the Sun at $0.28 \mathrm{AU}$. As part of this mission, the Extreme Ultraviolet Imager (EUI) will provide full-sun and high-resolution image sequences of the solar atmosphere at selected spectral emission lines in the extreme and vacuum ultraviolet.

To achieve the required scientific performances under the challenging constraints of the Solar Orbiter mission it was required to further develop existing technologies. As part of this development, and of its maturation of technology readiness, a set of breadboard and prototypes of critical subsystems have thus been realized to improve the overall instrument design.

The EUI instrument architecture, its major components and sub-systems are described with their driving constraints and the expected performances based on the breadboard and prototype results. The instrument verification and qualification plan will also be discussed. We present the thermal and mechanical model validation, the instrument test campaign with the structural-thermal model (STM), followed by the other instrument models in advance of the flight instrument manufacturing and AIT campaign.
\end{abstract}

Keywords: Extreme Ultraviolet Imager, Solar Orbiter, breadboard, prototype, instrument design, model philosophy, thermo-mechanical test, AIT

\section{INTRODUCTION}

The Solar Orbiter mission ${ }^{[1],[2],[3]}$ has been selected within the Cosmic Vision Science Program of European Space Agency (ESA). It will be launched in 2017 and is devoted to solar observation from an elliptical orbit around the Sun approaching to 60 solar radii. It will provide unprecedented close-up and high-latitude observations of the Sun.

The Extreme Ultraviolet Imager (EUI) instrument is one of the ten scientific instrument on-board the Solar Orbiter mission. It will provide image sequences of the solar atmospheric layers from the chromosphere into the corona and contribute to the following four outstanding Solar Orbiter science themes ${ }^{[4],[5],[6]}$ :

- What are the origins of the solar wind streams and the heliospheric magnetic field?

- What are the sources, acceleration mechanisms, and transport processes of solar energetic particles?

- How do coronal mass ejections evolve in the inner heliosphere?

- Explore, at all latitudes, the energetics, dynamics and fine-scale structure of the Sun's magnetized atmosphere

Space Telescopes and Instrumentation 2012: Ultraviolet to Gamma Ray,

edited by Tadayuki Takahashi, Stephen S. Murray, Jan-Willem A. den Herder,

Proc. of SPIE Vol. 8443, 844307 - (c) 2012 SPIE - CCC code: 0277-786/12/\$18 - doi: 10.1117/12.924343

Proc. of SPIE Vol. $8443844307-1$ 
The EUI instrument is composed of two high-resolution imager (HRI), one at the hydrogen Lyman- $\alpha$ line $\left(\mathrm{HRI}_{\text {Lya }}\right)^{[12]}$ and one in the extreme ultra-violet (EUV) at $174 \AA$ (HRI $\mathrm{HUV}_{\mathrm{EUV}}$ ); and one dual band full-sun imager (FSI) working alternatively at the two $174 \AA$ and $304 \AA$ passbands ${ }^{[7],[8]}$.

EUI is a compact instrument based on a passive thermo-mechanical design (no active control and passive detector cooling) taking advantage of a low CTE optical bench on which the three EUI channels are mounted and co-aligned. It takes heritage of the PROBA2-SWAP ${ }^{[10]}$ for the design of the HRI channels, of the entrance filters, and of the detectors and cameras. The FSI channel is based on a design derived from the HERSCHEL Rocket ${ }^{[11]}$. The EUV channels mirror coating are based on multilayers developed for SOHO-EIT, STEREO-EUVI EUV. Because of spacecraft constraints, a dedicated Common Electrical Box (CEB) unit provides the electrical interfaces with the platform. It also drives the mechanisms of the optical bench unit, and processes the images from the three channels. A very low telemetry allocation, due to the mission orbit, further requires a dedicated compression and on-board data processing, performed within the CEB.

To achieve the EUI performances, a set of new technologies have been developed ${ }^{[9]}$. As part of the instrument preliminary development phase, breadboards and prototypes have been developed and used to improve the instrument design and characterize the new technologies. In the present paper, we summarize the outcomes of this development phase. We also present the various instrument models that are now being manufactured for mechanical, thermal and electrical qualification and verifications that will be performed as part of an overall test plan.

\section{EUI INSTRUMENT DESCRIPTION}

\subsection{EUI on Solar Orbiter}

The EUI instrument is internally mounted in the Solar Orbiter platform. As the other remote sensing instruments, it is protected by a heat shield that limits the impact on the payload when approaching the Sun. At 60 solar radii, the heat input is indeed $\sim 13$ solar constants (i.e. $\sim 17 \mathrm{~kW} / \mathrm{m}^{2}$ ). Three small apertures in the heat shield are dedicated to the three EUI channels, as shown on Figure 1, allowing to image the Sun with limited impact on heat rejection.

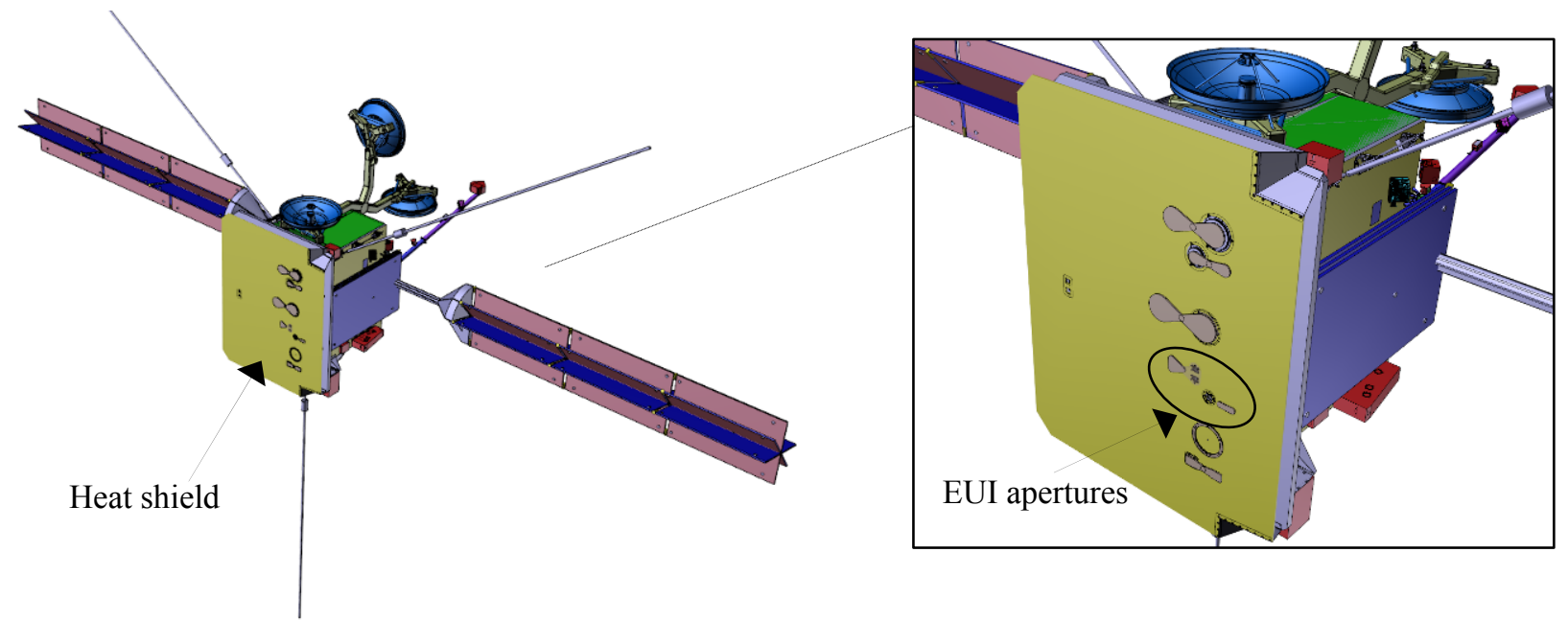

Figure 1 - EUI is an internally mounted instrument of Solar Orbiter. Its three channels observe the Sun through a heat shield that protects the spacecraft when it approaches the Sun.

\subsection{Channels overview}

The HRI channels use a two-mirrors Ritchey-Chrétien off-axis optical system, and the FSI uses a single mirror off-axis Herschelian system ${ }^{[4]}$. The normal-incidence telescopes are in co-alignment and operate independently. Their mirrors have optimized coatings for FUV or EUV reflectivity in each passband. The spectral selection is complemented with filters and filter wheel (in the FSI and $\mathrm{HRI}_{\mathrm{EUV}}$ channels) rejecting the visible and infrared radiation.

As shown on Figure 2, the HRI and FSI images are produced by respectively a two-mirror and a one-mirror telescope, working in near normal incidence. The EUV reflectivity of the optical surfaces obtained with specific EUV multilayered 
coating provides the spectral selection, complemented by filters rejecting the visible and IR radiation. The UV photons reach the detectors (back-thinned APS of $10 \mu \mathrm{m} 2 \mathrm{k} \times 2 \mathrm{k}$ for the HRI channels and $10 \mu \mathrm{m} \mathrm{3k}$ x 3k for the FSI channel) where they are converted into an electrical signal in front-end electronics (FEE), before being compressed and stored in the CEB (common electronic box). For each detector pixel, the resulting signal is proportional to the solar flux corresponding to the small viewing angle of the pixel in the given passband.
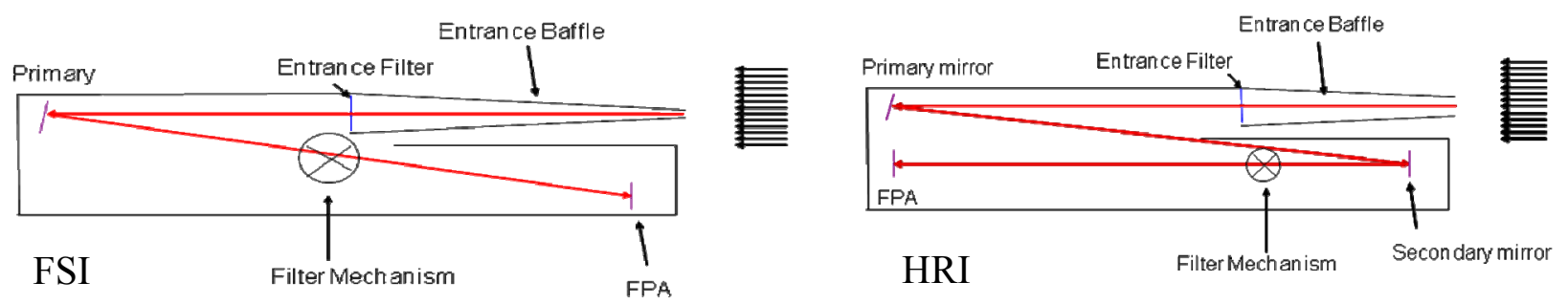

Figure 2 - Schematic layouts of the FSI (left) and HRI (right) channels

Table 1 lists the intended temporal and spatial resolutions, field-of-view (FOV) as well as passbands of the EUI instrument. As shown on Figure 3, the HRI channels will image a central area of the solar disk, with a field of view corresponding to $15 \%$ of the solar diameter, with a $200 \mathrm{~km} /$ pixel resolution (at the perihelion), equivalent to a 0.16 arcsec resolution at 1 Astronomical Unit (AU). The FSI channel field of view will cover the equivalent of two solar diameters with a resolution of $1800 \mathrm{~km} /$ pixel.

Table 1 - Main parameters of the three EUI channels (FSI, HRI $\mathrm{EUV}_{\text {av }}$ and $\mathrm{HRI}_{\mathrm{Lya}}$ )

\begin{tabular}{|c|c|c|}
\hline Channel & Parameter & Values \\
\hline FSI & $\begin{array}{l}\text { Passband } \\
\text { FOV } \\
\text { Resolution (2 px) } \\
\text { Cadence }\end{array}$ & $\begin{array}{l}17.4 \mathrm{~nm} \& 30.4 \mathrm{~nm} \\
3.8 \operatorname{arcdeg} \\
9 \operatorname{arcsec} \\
600 \mathrm{~s} \\
\end{array}$ \\
\hline HRI $_{\text {EUV }}$ & $\begin{array}{l}\text { Passband } \\
\text { FOV } \\
\text { Resolution (2 px) } \\
\text { Cadence } \\
\end{array}$ & $\begin{array}{l}17.4 \mathrm{~nm} \\
0.28 \text { arcdeg } \\
1 \text { arcsec } \\
\geq 1 \mathrm{~s} \\
\end{array}$ \\
\hline HRI $_{\text {Lya }}$ & $\begin{array}{l}\text { Passband } \\
\text { FOV } \\
\text { Resolution (2 px) } \\
\text { Cadence }\end{array}$ & $\begin{array}{l}121.6 \mathrm{~nm} \\
0.28 \operatorname{arcdeg} \\
1 \operatorname{arcsec} \\
\leq 1 \mathrm{~s}\end{array}$ \\
\hline
\end{tabular}
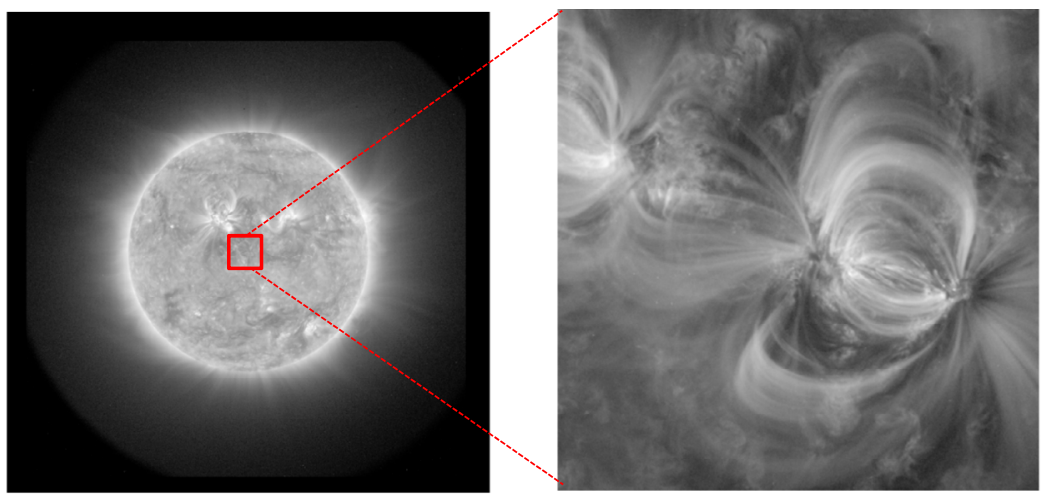

Figure 3 - The FSI (left) and HRI (right) field of view. FSI provides a full sun disk image and HRI provides a high resolution imaging of the disk center 


\subsection{The two EUI units}

The three EUI channels are mounted in an Optical Bench Structure (OBS) unit made out of a CFRP sandwich panel with an aluminum honeycomb core optimized for opto-mechanical and thermal stability. For each channel, a set of subsystems is mounted on the OBS: the FSI and HRI mirrors, the FSI and HRI $\mathrm{EUV}_{\mathrm{EUV}}$ filter wheels, a high voltage unit for the intensified Ly- $\alpha$ camera, one door, one entrance baffle and one camera (called Focal Plane Assembly FPA) per channel (Figure 4).

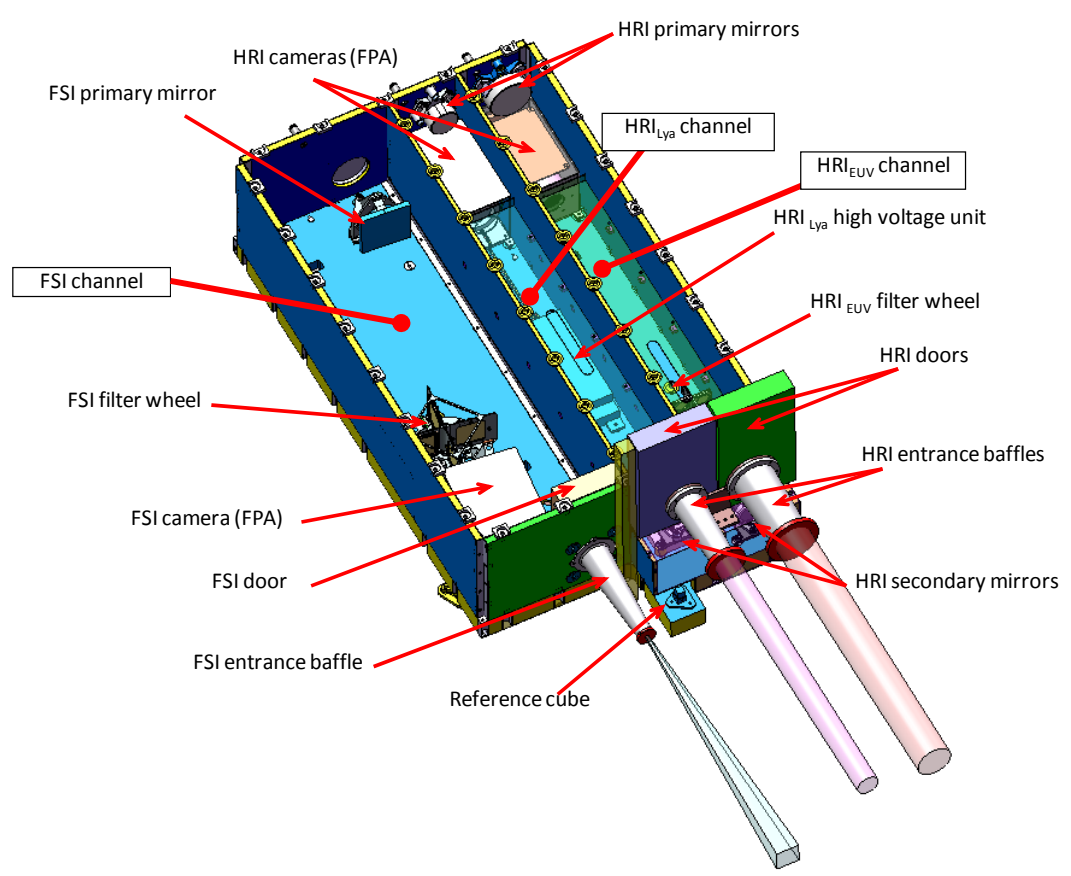

Figure 4 - The three EUI channels and their sub-systems are mounted on an Optical Bench Structure (OBS) unit.

A Common Electronics Box (CEB) unit provides the control of the OBS unit mechanisms (filter wheels and doors), contains the image processing, and ensures the electrical interface with the spacecraft on-board computer and power supply. The CEB is composed of a processor board (that contains the CPU and provides the digital interface with the spacecraft), a compression board (that connects the CEB with the three OBS cameras and contains a compression device), a power board (that houses four secondary power supply units, one for the CEB and one each for the three cameras) and an auxiliary board (that connects to the OBS auxiliary functions as temperature sensors, motor drive and position sensing, heaters and control the high voltage power supply). The CEB is also running onboard software that ensures the interface between the camera and the CEB, and between the CEB and the S/C.

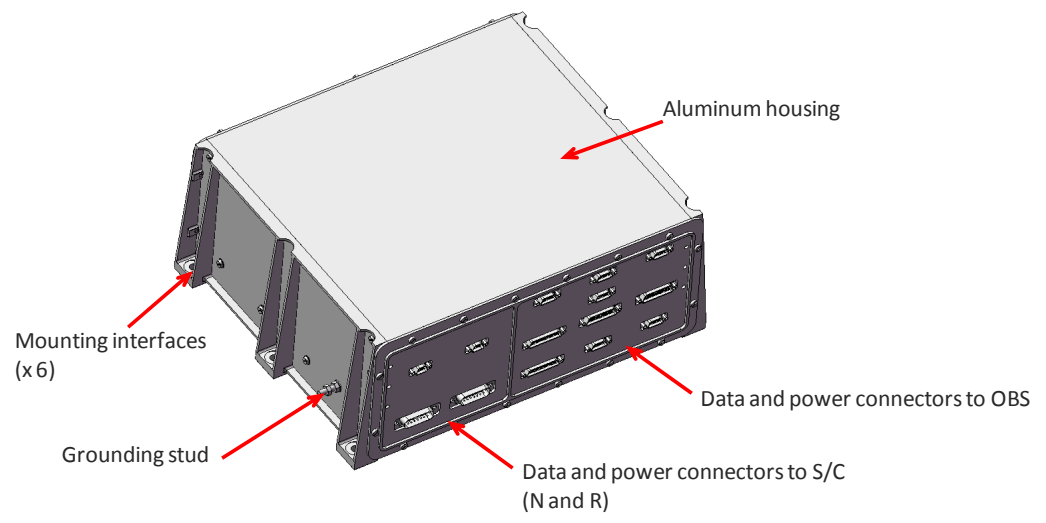

Figure 5 - A Common Electronic Box (CEB) unit provides electrical interfaces with the Spacecraft, and drives the OBS sub-systems. It also provides data processing, including image compression. 


\section{TECHNOLOGICAL DEVELOPMENT}

Prototypes have been developed to validate the following four EUI key technologies:

- The HRI entrance baffle, whose rejection limiting the heat input from the Sun

- The EUV entrance filters, located at end of the entrance baffles, which have to survive launch but also the high solar flux input.

- The EUV mirror coatings, whose reflectivity contributes to spectral purity

- The Active Pixel Sensor (APS) detector, providing a high dynamic range with low power consumption

\subsection{The HRI entrance baffle}

The entrance baffle of each EUI channel is the mechanical part located between the entrance pupil and the entrance filter.

The HRI entrance baffles are reflective inside. They are designed to reject a maximum of the incoming sunlight out of the instrument, thereby reducing the heat load. The remaining heat load absorbed by the entrance baffles, and by its associated entrance filter, is evacuated to space via a $\mathrm{S} / \mathrm{C}$ radiator. The FSI entrance baffle is black inside, and simply absorbs the low power incoming through its small entrance pupil.

A dedicated development plan has been established; the flow diagram is shown in Figure 6.

The first agreement of this development was the very good correlation between measured HRI entrance baffle prototype rejection with the theoretical ray-tracing computations (Figure 8).The corresponding test configuration is shown on Figure 7. By injecting a known collimated flux at the baffle entrance and measuring the output at the same entrance (by use of an integrating sphere), the baffle global rejection can be characterized for various incidence angles.

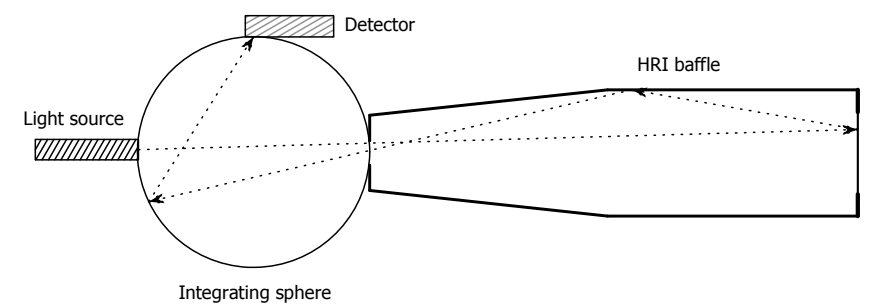

Figure 7 - Optical test setup of the HRI entrance baffle prototype

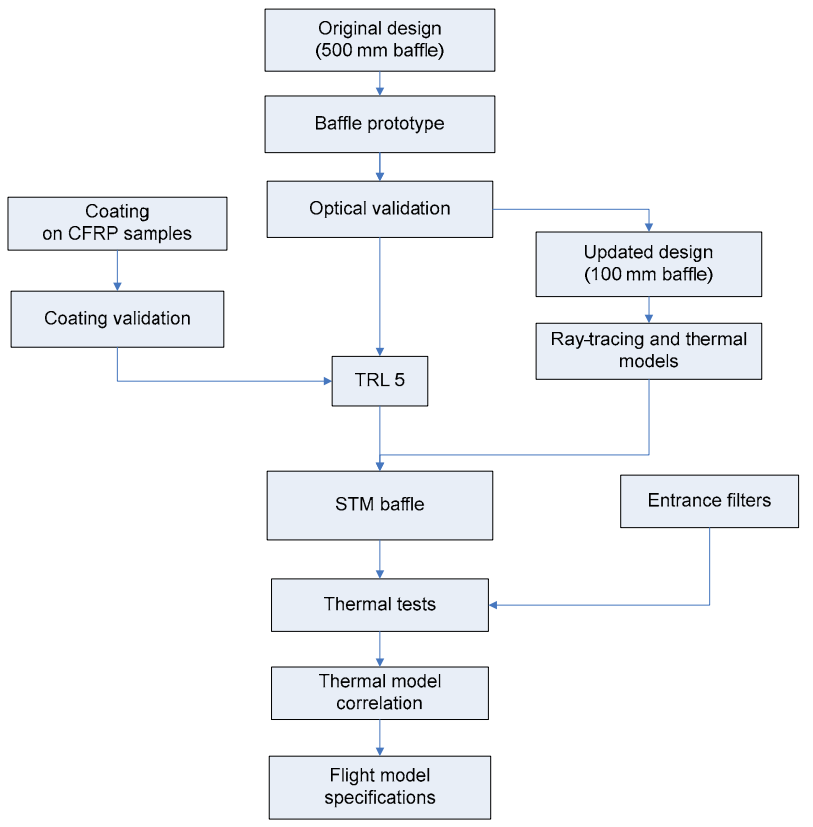

Figure 6 - Entrance baffle development flow

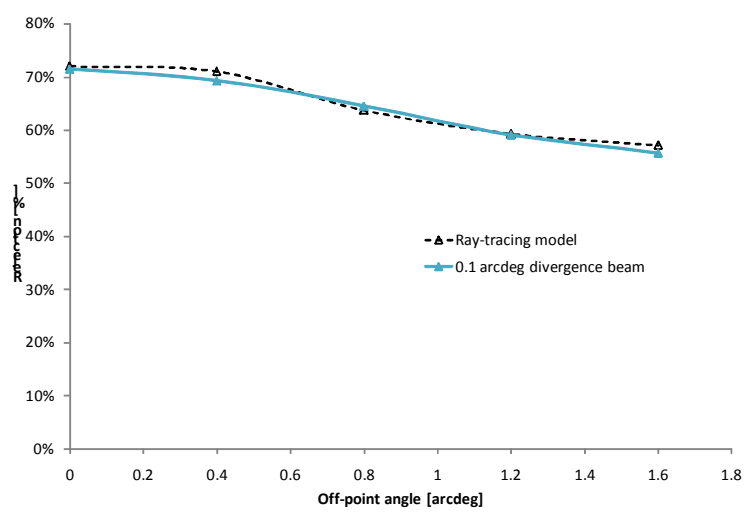

Figure 8 - Entrance baffle measured optical rejection

The second result of this development is the validation of the use of aluminum coating on CFRP layers. The HRI entrance baffles must indeed be light-weight because of the overall EUI mass budget and CFRP is a potential material candidate for these entrance baffles. Manufacturing a CFRP tube is not complex, but to achieve the design internal surface finish and thermo-optical properties, a reflective specular coating must be applied. A dedicated aluminum 
coating process has been developed and applied on CFRP samples. The reflectivity of these coatings has been measured with and without a protective layer (Figure 9), and before/after thermal cycling (Figure 10).

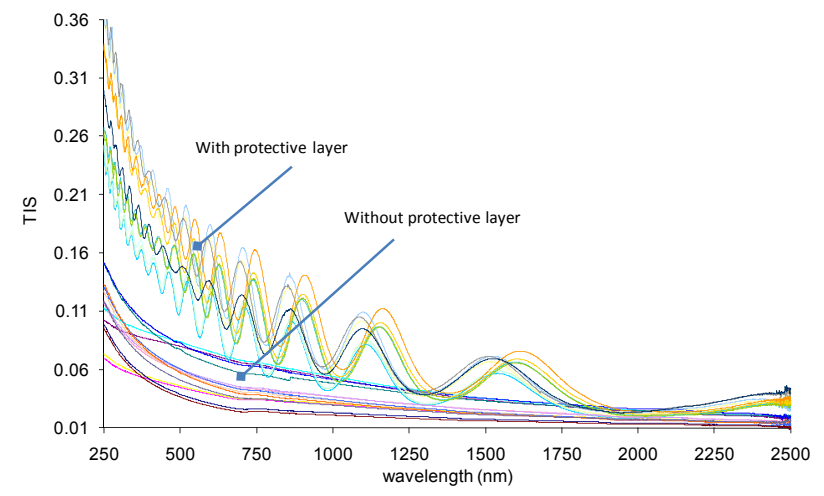

Figure 9 - Total Integrate Scatter (TIS) of coated CFRP samples with and without protective layer.

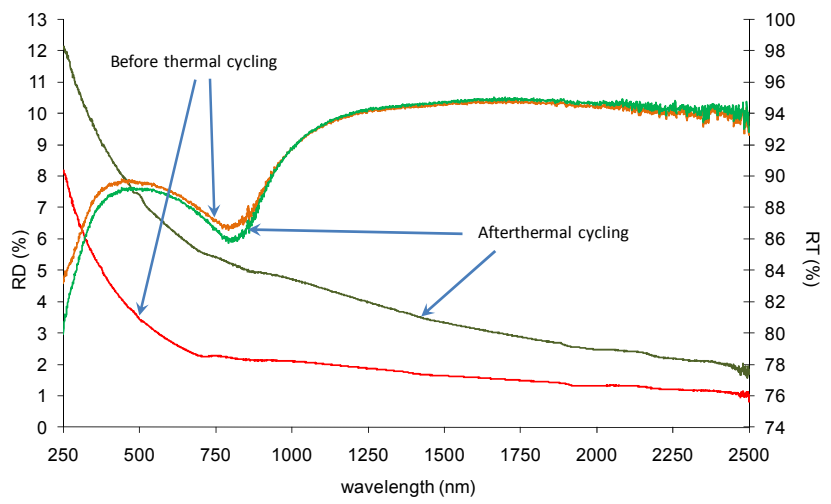

Figure 10 - Total and diffuse reflectivities of coated CFRP samples (here without protective layer).

The HRI entrance baffle design has been, since these tests, adapted for mechanical constraints of the OBS structure unit on which they are mounted. Their length has been reduced to $100 \mathrm{~mm}$ with a conical heat rejection mirror. This latest design has thus been optically and thermally modeled with the spacecraft feedthrough, which surround the channel unobstructed field of views (UFOV) within the heat shield, to determine the optimum feedthrough vane geometry to limit potential straylight and to quantify its infrared contribution on the baffle thermal balance.
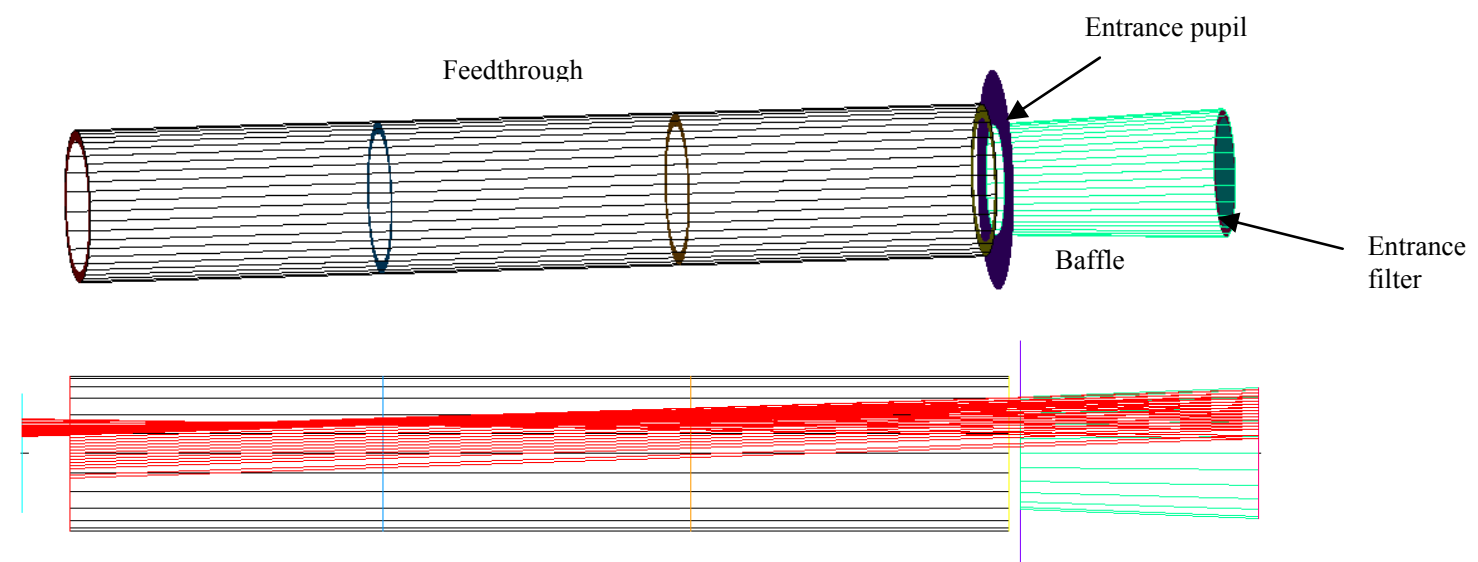

Figure 11 - Ray-tracing model of the heat shield feedthrough, located in front of the EUI entrance baffle.

\subsection{The EUV filters}

At back of the entrance baffle is located an entrance filter that provides a first spectral selection and suppresses most of the UV, visible and IR counterparts of the solar radiation, which is of primary importance to avoid over-heating the instrument cavity. For $\mathrm{HRI}_{\mathrm{Lya}}$ the entrance filter is a MgF2 surface coated with aluminum. For the $\mathrm{HRI}_{\mathrm{EUv}}$ and the FSI channels, entrance filters are thin aluminum (Al) foils (150 nm thickness).

Focal filters are also included in the $\mathrm{HRI}_{\mathrm{EUV}}$ and FSI filter wheels. In FSI, the filters are $\mathrm{Al} / \mathrm{Zr}$ for the short wavelengths and $\mathrm{Al} / \mathrm{Mg} / \mathrm{Al}$ for the longer wavelengths. In $\mathrm{HRI}_{\mathrm{EUV}}$, the filters are $\mathrm{Al}$ filters.

All these filters are available commercially and have long flight heritage. However it appears that a specially designed EUV entrance filter is required to sustain the high thermal load the entrance filter will have to endure, but also the large FSI focal filters that have to survive launch mechanical loads. A dedicated development plan has thus been established for these EUV filters. 


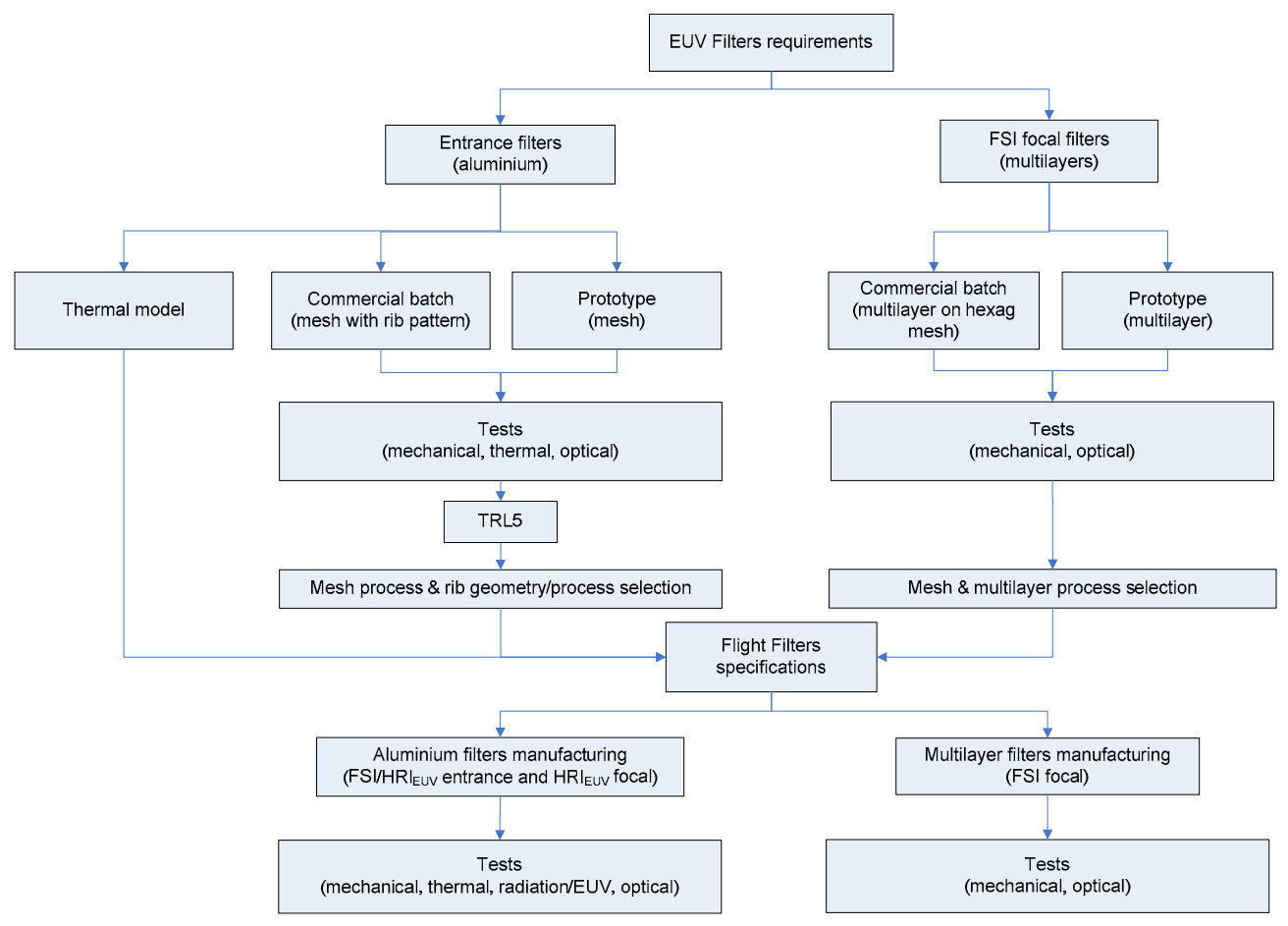

Figure 12 - Development flow of the EUV filters for both the $\mathrm{HRI}_{\mathrm{EUV}}$ entrance filter and the FSI focal filters.

A set of commercial Al filters (Figure 13) with various mesh properties has been used to validate the thermo-optical model. Thermal and mechanical tests have also been performed to determine which type of mesh is best suited for launch and can withstand the high solar flux input (13 solar constants). In parallel, custom filter prototypes have been developed to validate an improved mesh conductivity based on the Ni mesh grown on top of a $150 \mathrm{~nm}$ aluminum layer (Figure 14).
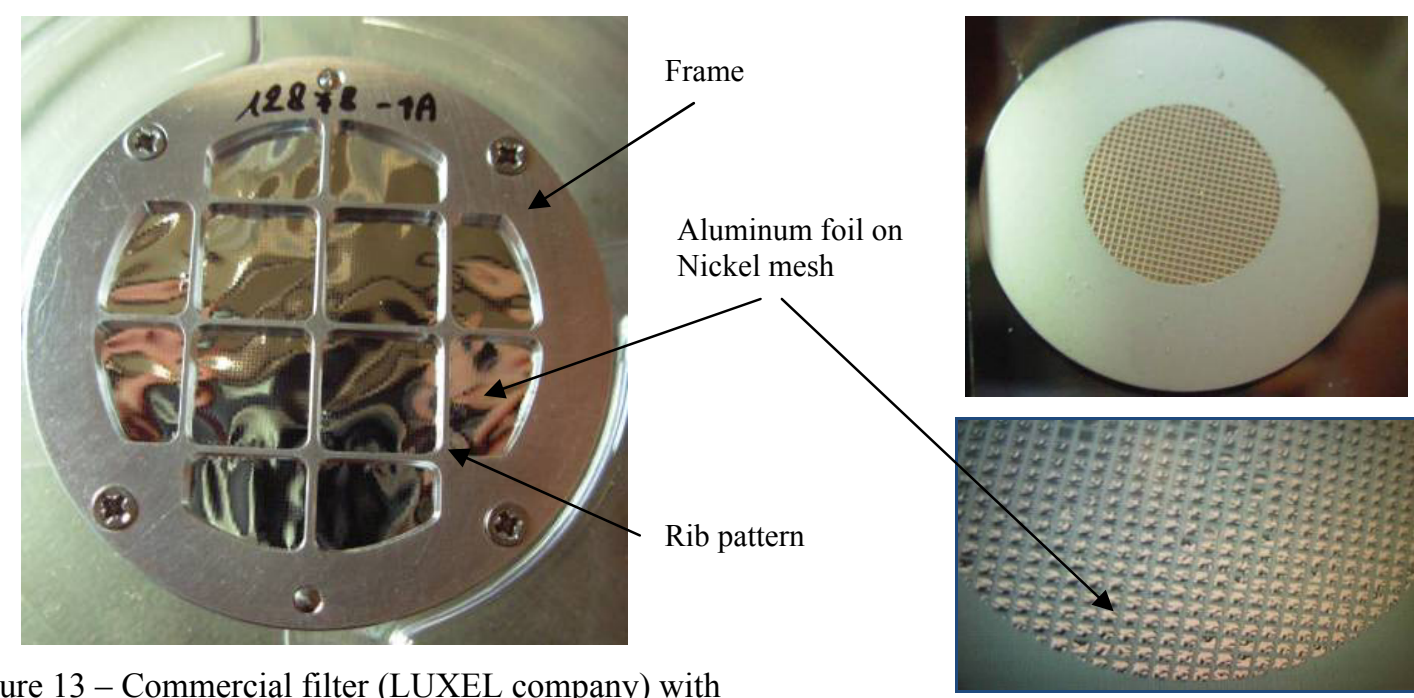

Figure 13 - Commercial filter (LUXEL company) with reinforced frame to increase thermal conductivity. The mesh pattern is optimized for mechanical and thermal resistance.

Figure 14 - Prototype Ni mesh grid pattern grown on an Al foil, made at the Centre Spatial de Liège (CSL).

- Thermal tests

The thermal test is performed in a $1.5 \mathrm{~m}$ vacuum chamber test facility of the CSL with a thermal shroud to provide a cold environment around filter (Figure 15). The heat flux input (up to $13 \mathrm{SC}$ with $10 \%$ homogeneity) is provided by 
an illumination system located outside of the chamber. It is composed of a Xenon lamp whose light is collimated with a lens located in front of a fused silica vacuum window allowing the beam to enter the system.

An infrared camera is used to record the filter temperature. Such camera cannot be used under vacuum and is placed in a canister with a $\mathrm{ZnSe}$ window (thermal IR transparent glass) located within the vacuum chamber. A set of temperature sensors and heaters also allows to measure parasitic conductive fluxes. The IR camera signal has indeed three contributions, only one of them representing the actual filter temperature: the self-emitted radiation from the filter (signal), the environment radiation reflected by the filter (noise) and the emission from the atmosphere surrounding the filter (noise). The atmospheric emission is mostly attenuated by performing the test under vacuum. The filter emissivity being low - about 5\% - the environment reflection contribution is high and must be kept below the intrinsic emission of the filter, which is the desired signal. Hence, the thermal shrouds were fed with liquid nitrogen $(80 \mathrm{~K})$ to minimize this contribution. A reference filter is used to calibrate the camera. This reference filter is outside of the input light beam and its temperature is controlled by heaters. During the test, its temperature is increased to reach the same temperature as the filter under test and calibrate the camera.

\section{- Mechanical tests}

The mechanical test consists of applying a gradually increased pressure on one side of the filter and measuring its deflection versus the pressure. The theoretical deflection is calculated by considering the mesh grid as the thin plate and the Ni Young's modulus.

The major result of these tests is that one of the commercial filters has a mesh pattern that provides sufficient conductivity and mechanical resistance to survive the $13 \mathrm{SC}$ thermal load and the launch mechanical environment.
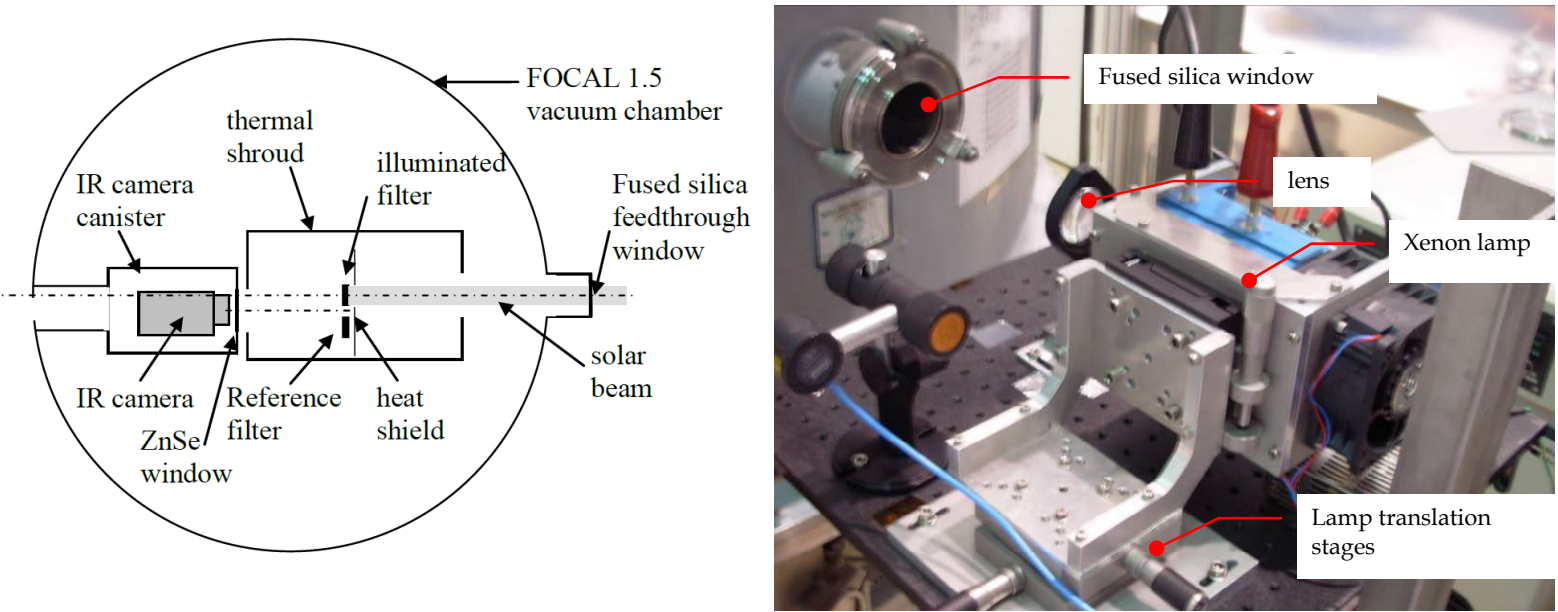

Figure 15 - Thermal test setup used to measure the Al filter thermal conductivity and resistance to input of $13 \mathrm{SC}$.

A set of commercial FSI focal filters (see Figure 16) have also undergone a series of tests including an EUV transmittance measurement (Figure 17) and a mechanical validation similar to the Al filters one.
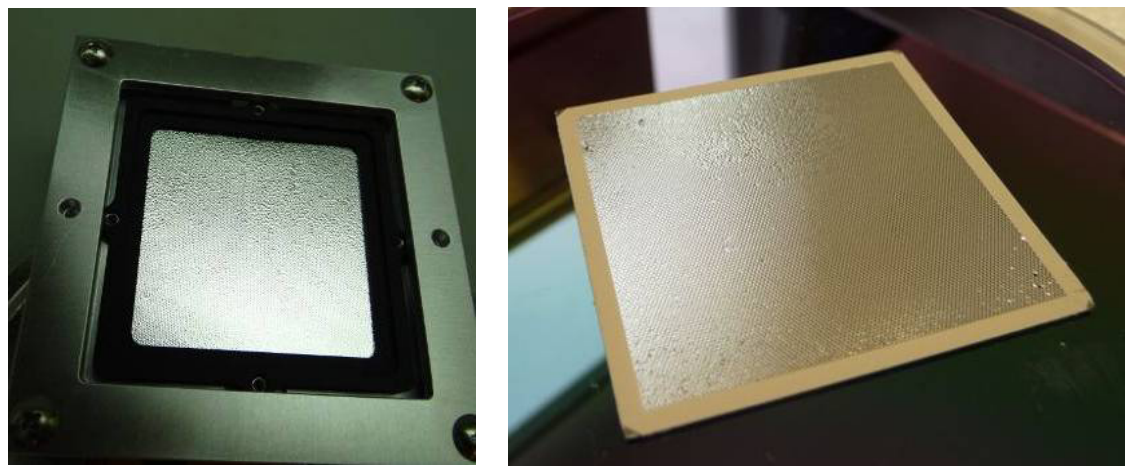

Figure 16 - Commercial (LUXEL) and prototype FSI focal filter $(40$ x $40 \mathrm{~mm})$ with hexagonal Nickel mesh pattern. 

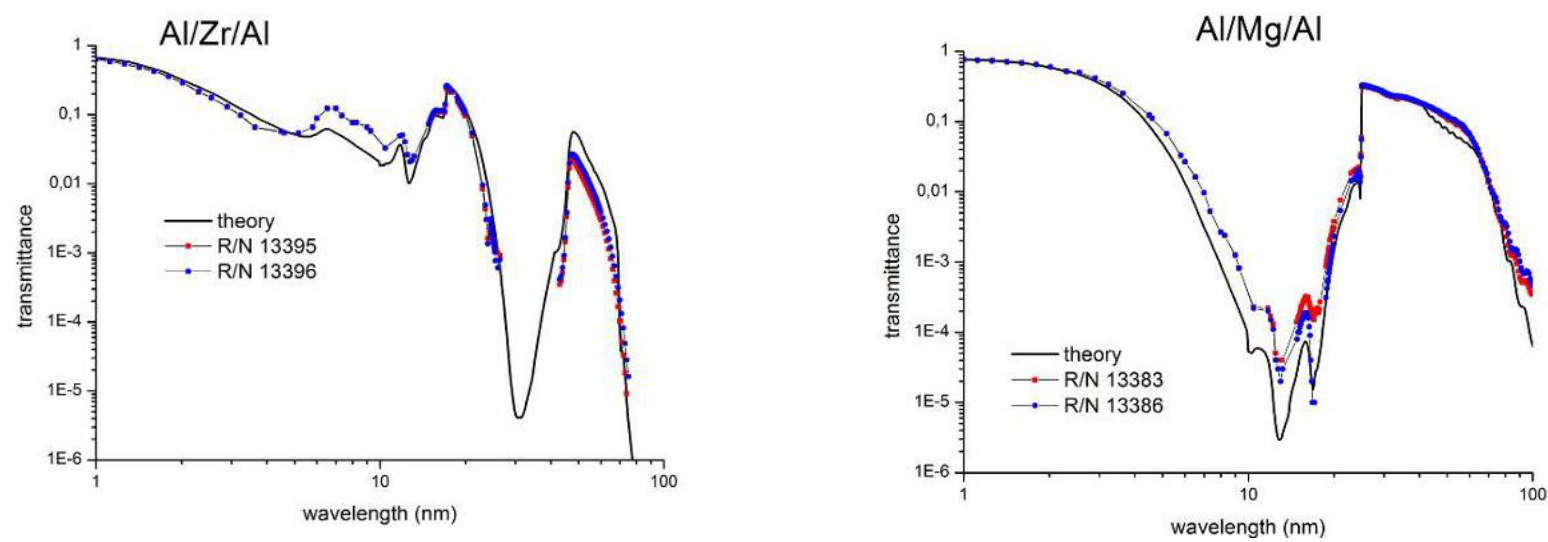

Figure 17 - Measured transmittance of FSI focal filters prototypes (Top: Al/Zr, Bottom: Al/Mg)

\subsection{The EUV multilayer coatings}

The EUV channels make use of innovative $\mathrm{Al} / \mathrm{Mo} / \mathrm{B} 4 \mathrm{C}$ multilayer coatings to improve the reflectivity. For the FSI channel this coating is furthermore tuned to allow the reflection of two bands centered on $174 \AA$ (Fe X) and $304 \AA$ (He II), and the rejection of several bright unwanted spectral lines (e.g. Fe XII $195 \AA$ and Fe XVI $335 \AA$ ).

A set of mirror coating prototypes were made on mirror samples (not flight shape and size representative as the process is similar and no change is expected when going to flight-like dimensions) as part of the dedicated development flow shown in Figure 18.

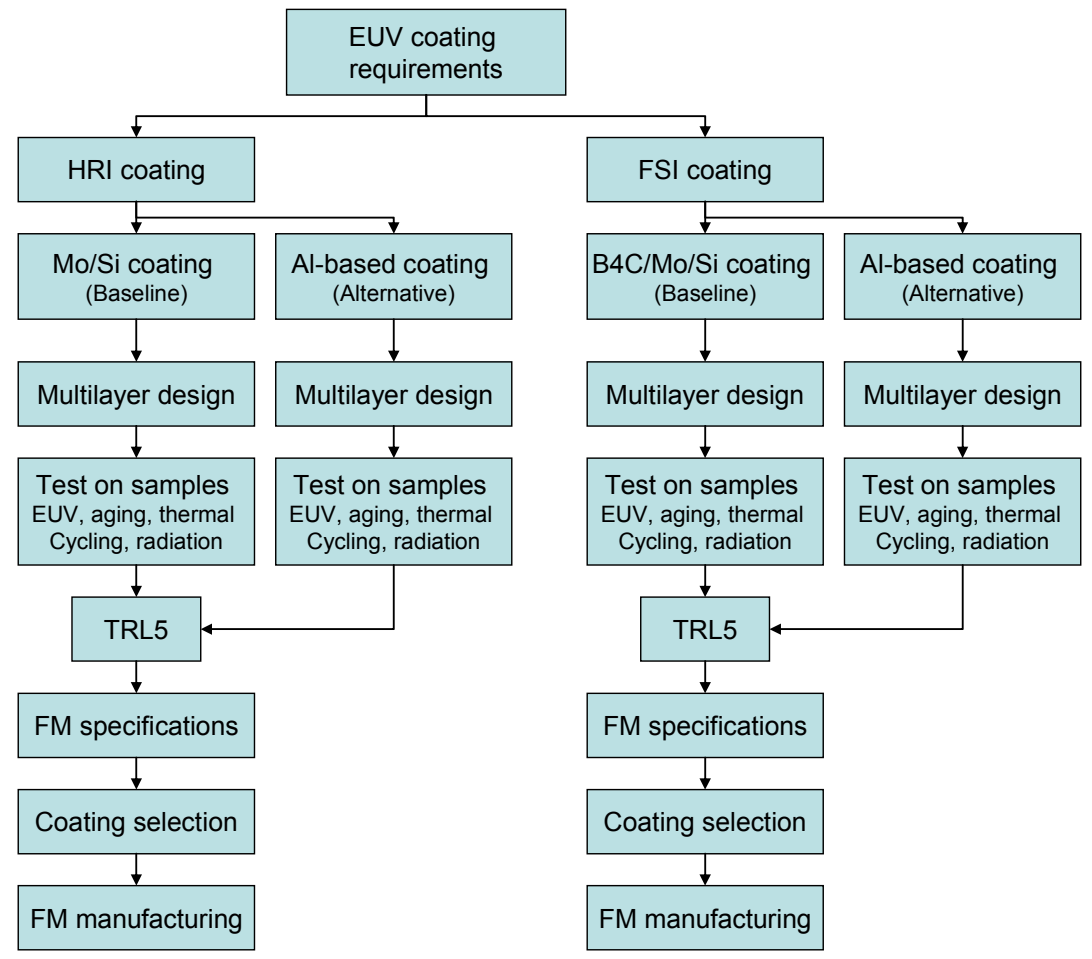

Figure 18 - Development flow diagram of the EUV mirror multilayer coatings.

$\mathrm{Al} / \mathrm{Mo} / \mathrm{B} 4 \mathrm{C}$ multilayers prototypes were subjected to an extensive ageing study. The coatings make use of barrier layers to stabilize the multilayer structure. Thermal cycling between $-50{ }^{\circ} \mathrm{C}$ and $+70{ }^{\circ} \mathrm{C}$ and exposition to warm water vapor have demonstrated that these new multilayers are very stable and should not vary during the mission. These studies will be complemented by irradiation tests under proton and neutron beams to ensure that the optics will not degrade during the mission lifetime. 
Single band coatings have been fabricated and reflectivities up to $55 \%$ at $17.1 \mathrm{~nm}$ were reached. This new coating will undergo extensive environmental tests to validate their structural stability before they become the baseline for the HRI $_{\text {EUV. }}$.

\subsection{The detectors}

The EUI telescopes use large-format CMOS radiation-hardened active-pixel image sensor (APS). For the EUV channels, backside illuminated, thinned APS, sensitive in the EUV is considered as baseline. For the Ly- $\alpha$ channel, a front side illuminated, sensitive in the visible (VIS), is considered as baseline.

These detectors must have a low readout noise to allow shutterless operation, but also have a greater radiation tolerance (no charge transfer), low power consumption, operational temperature of $-40{ }^{\circ} \mathrm{C}$, high speed and dynamic range, non destructive readout, noise reduction and smart camera possibilities (e.g. random addressing, windowing, antiblooming...).

The development flow of the detectors (up to FM manufacturing) is shown in Figure 19. Based on science specifications, a back and a front side version of an APS detector prototype have been developed and characterized.

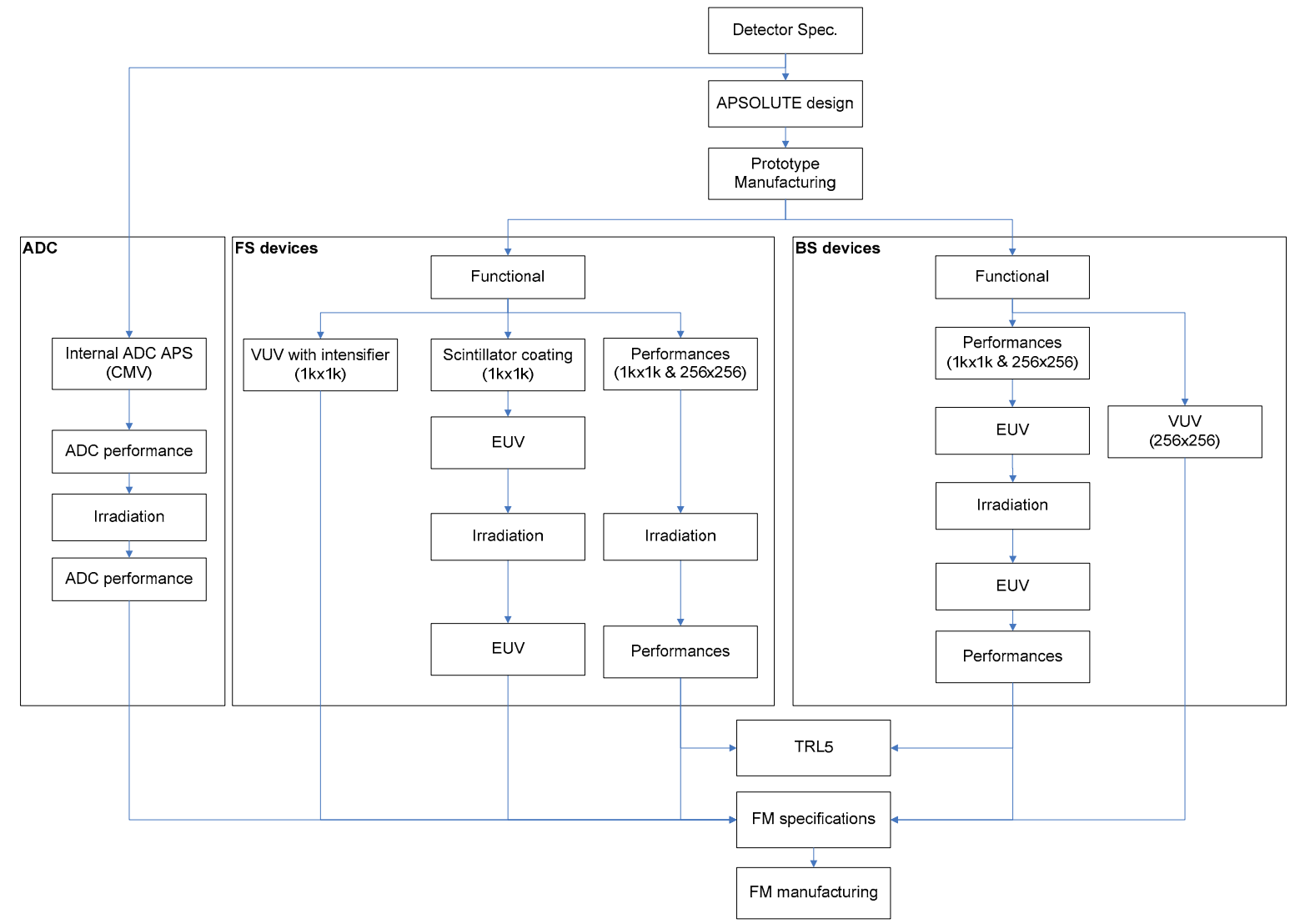

Figure 19 - Development flow of the EUI CMOS APS detectors.

The EUI detector prototypes were named APSOLUTE for APS Optimized for Low-noise and Ultraviolet Tests and Experiments. Two APSOLUTE image sensors were designed (Figure 20) and procured by the CMOSIS company:

- A 256 x 256 pixel array, containing 16 test pixel variants, organized in blocks of 64 x 64 pixels to select the best pixel design variant to be used in later flight devices.

- A 1024 x 1024 pixel array, containing the best guess pixel variant out of the 16 variants. Its size is close to the flight model size to validate manufacturing process (in particular back thinning) and large-scale performances.

In both cases, the pixels (and the rolling shutter) are controlled by off-chip signaling through the pixel control block. 
To achieve the requested high dynamic range, two gain paths are available per pixel. This results in $2 \times \mathrm{N}$ outputs per line of $\mathrm{N}$ pixels. $2 \times \mathrm{N}$ column gain stages are implemented.

Each column multiplexer sends $2 \times \mathrm{N}$ data to a single analog output channel. The output stage converts the signal to a fully-differential signal which can be sent to an off-chip A/D converter. An SPI interface is used to control various sensor settings (bias currents, voltage levels, gains ...) and a temperature sensor is read out over the SPI interface.

The prototype detectors were tested in a dedicated EGSE for powering and acquiring images (Figure 21). The tests that were conducted include flat field and MTF measurement, dark current characterization versus temperature, photon transfer curve (PTC) characterization (Figure 22), EUV response (Figure 23). These tests were performed before and after radiation hardness tests, i.e. Total Ionizing Dose (TID), Heavy Ions (HI) and Protons irradiation.

A possible back-up solution has also been tested. It is a front side illuminated devices with a dedicated scintillator coating. Such coating was used previously in camera of the SWAP instrument on PROBA2 ${ }^{[10]}$. The scintillator coating converts EUV photons to visible light detected by the front-side device.
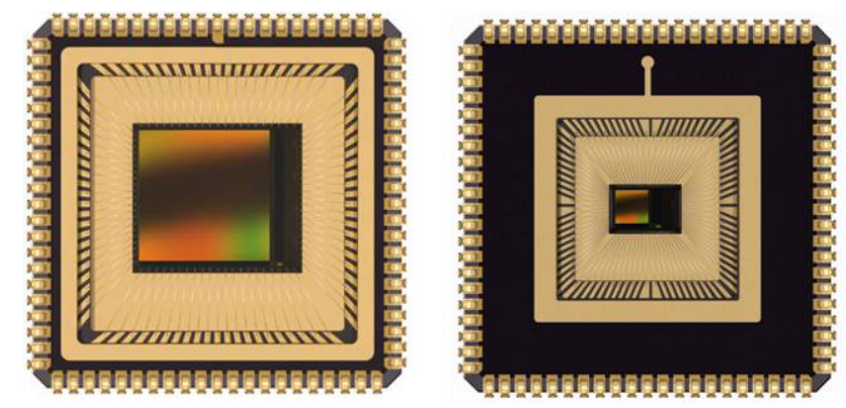

Figure 20 - APS prototypes developed to validate the EUI detector baseline.

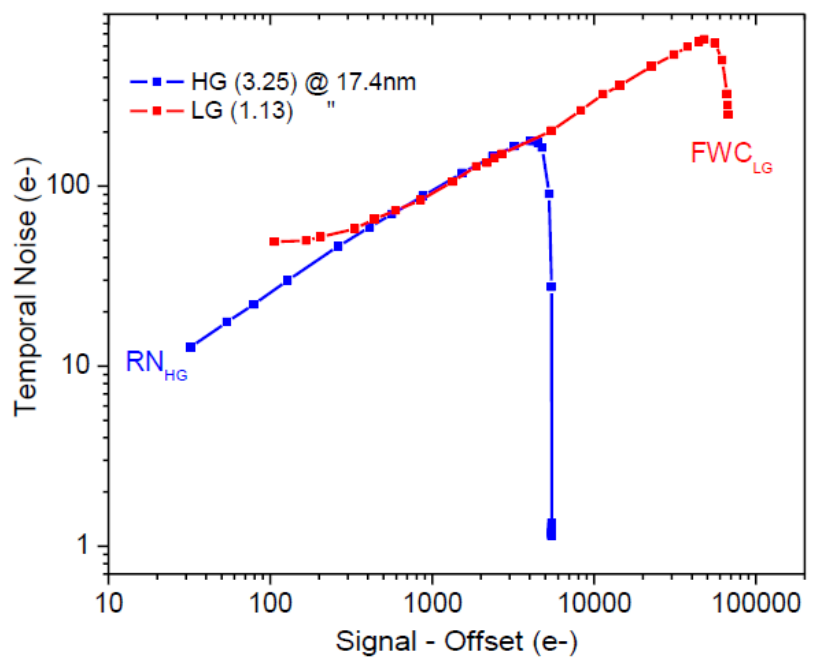

Figure 22 - PTC measured at $17.4 \mathrm{~nm}$, for high and low gains, on a $1 \mathrm{k} \mathrm{x} 1 \mathrm{k}$ back-side prototype.

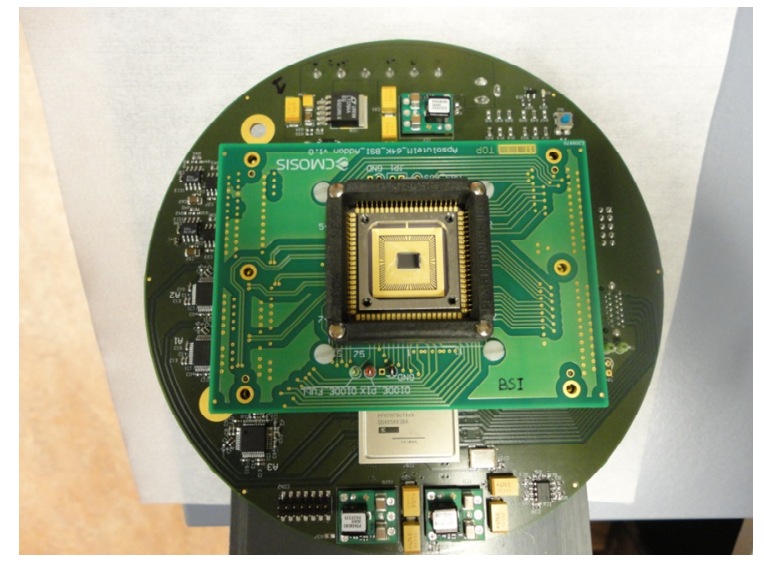

Figure 21 -APSOLUTE EGSE: architecture (left) and picture with an a prototype device integrated (right)

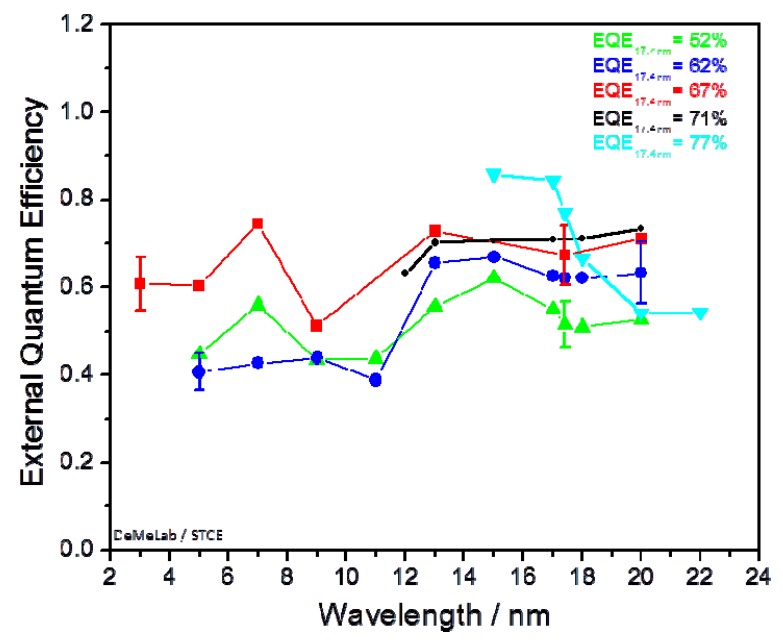

Figure 23 - Measured detector response in the EUV wavelengths of EUI APS detector prototypes.

The complete sequence demonstrated the suitability of the CMOS-APS technology as the baseline detector and reaching the required qualification status (i.e. a radiation tolerant APS detector with very low noise, large dynamic range and EUV sensitivity). Table 2 summarizes the detector prototype test results, as compared with EUI detector requirements. 
Table 2 - APSOLUTE detectors test results as compared with EUI detector requirements

\begin{tabular}{|l|c|l|}
\hline \multicolumn{1}{|c|}{ Parameter } & Flight Requirement & \multicolumn{1}{c|}{ Prototype results } \\
\hline Number of pixels & $\begin{array}{c}2 \mathrm{k} \times 2 \mathrm{k}(\mathrm{HRI}) \\
3 \mathrm{k} \times 3 \mathrm{k}(\mathrm{FSI})\end{array}$ & $1 \mathrm{k} \times 1 \mathrm{k}$ and $256 \times 256$ \\
\hline Pixel pitch & $10 \mu \mathrm{m}$ & $10 \mu \mathrm{m}$ \\
\hline Spectral range & $\begin{array}{c}10-40 \mathrm{~nm}(\mathrm{EUV}) \\
121 \mathrm{~nm}(\mathrm{Ly}-\mathrm{a})\end{array}$ & Measured $5-20 \mathrm{~nm}$ and $325-1025 \mathrm{~nm}$. \\
\hline Maximum pixel rate (frame rate) & $10 \mathrm{FPS}$ & $10 \mathrm{FPS}$ \\
\hline MTF / optical crosstalk & $50 \% / 5 \%$ & $>50 \%$ (in visible) \\
\hline Sensitivity (detected ph/incident ph) & $>50 \%$ & $>50 \%$ at $17.4 \mathrm{~nm}$ \\
\hline Readout noise & $<5 \mathrm{e}-\mathrm{RMS}$ & $<4 \mathrm{e}$ (on back-side devices) \\
\hline Average dark signal & $10 \mathrm{e}-/ \mathrm{s} / \mathrm{px}$ & Achieved at $-40{ }^{\circ} \mathrm{C}$. \\
\hline Pixel saturation charge & $>80 \mathrm{ke}-$ & Up to $83 \mathrm{ke}-$ at linear full well capacity (on back-side) \\
\hline Non-linearity (p-p) & $<2 \%$ & $<2 \%$ \\
\hline Power consumption & $<0.5 \mathrm{~W}$ & $\sim 0.1 \mathrm{~W}$ \\
\hline Radiation hardness & $>30 \mathrm{kRad}$ & $>100 \mathrm{kRad}$ (on back-side) \\
\hline Operational T ${ }^{\circ}$ range & $-60 /+60{ }^{\circ} \mathrm{C}$ & Tested from $-60{ }^{\circ} \mathrm{C}$ to $+60{ }^{\circ} \mathrm{C}$ \\
\hline
\end{tabular}

\section{BREADBOARDS}

In addition to the prototypes developed for the key technologies, a breadboard of the following sub-units has been built to validate their preliminary design and improve the fidelity of the instrument resource requirements (power/mass):

- The CEB, which provides electrical interfaces with the platform, drives the mechanisms and processes the images from the three channels.

- The FEE, which is the detector proximity electronic, and provides interface with the CEB.

- The internal doors, which provide a contamination protection during on-ground activities and launch, and allow inflight protection of the channels when the instrument is not in operation.

- The multi-channel plate intensifier, which is part of the $\mathrm{HRI}_{\text {Lya }}$ channel camera, and its high voltage power supply

\subsection{Common Electronic Box (CEB)}

The following breadboards (Figure 24) have been implemented as part of the CEB boards (processor board, compression board, power supply board, auxiliary board). A breadboard of the on-board software has also been developed.

- $\quad$ Power conditioning

The CEB supplies power conversion and conditioning for the CEB and the three imagers. Two power converter types have been developed in order to determine overall power supply efficiency, one for the CEB and one for the three imagers (common design). In addition to this point of load (POL) converters were developed to determine the efficiency achievable.

- Processor

To control the EUI instrument, a breadboard of interface to the spacecraft and perform science processing requirements with a UT699 processor has been implemented. To interface the CPU to the instrument and control the Data queues (see Section 0) an FPGA has been used.

- Detector control and motor drivers (auxiliary board)

There are five motors on the EUI OBS unit, three to control the doors and two to control the filter wheels.

To prove the micro stepping algorithms and analogue drive implementation is compatible with the door and filter wheels, a PCB of the drive electronics has been developed which proves the power switching circuitry. This board interfaces to an EGSE board housing an FPGA in which sequencing logic will be developed to prove the stepping 
algorithms (this sequencing logic will eventually be included in the processor board FPGA). This breadboard is planned to be tested with the door and filter wheel breadboards.

- Data queues (auxiliary board)

To support science requirements data queues are implemented in the CEB. There are two queues, one called the 'one hour queue' and the second the 'spacecraft queue'. The present sizes of these are 4 and 2 Gbytes respectively. The breadboard includes an SDRAM controller with scrubbing and EDAC correction, implementing one bank of representative flight devices with further memory implemented using commercial memory. The breadboard plans implements a non-flight packaged version of the FLASH memory module, in which the SDRAM controller is implemented.

\section{- Compression}

To meet the science requirements a large amount of processing and compression is needed on the output data of the imagers. Pre-processing is performed by logic in an FPGA and the compression performed by a 'compression system' afterwards. The data is then passed onto the processor board.

The compression breadboard demonstrates that the data rate throughput can meet the pixel pre-processing specifications and shows the FPGA resources are sufficient to meet the present science requirements. The breadboard supplies connectors so that a piggy back daughter board can be added which contains a compression chip to be selected. The plan is test the system with one off the shelf compression systems.

\section{- $\quad$ EGSE}

In addition to the CEB breadboards, an EGSE for High speed link was used to aid testing of the Compression board, supporting the CEB internal interfaces between the Compression and Processor board.
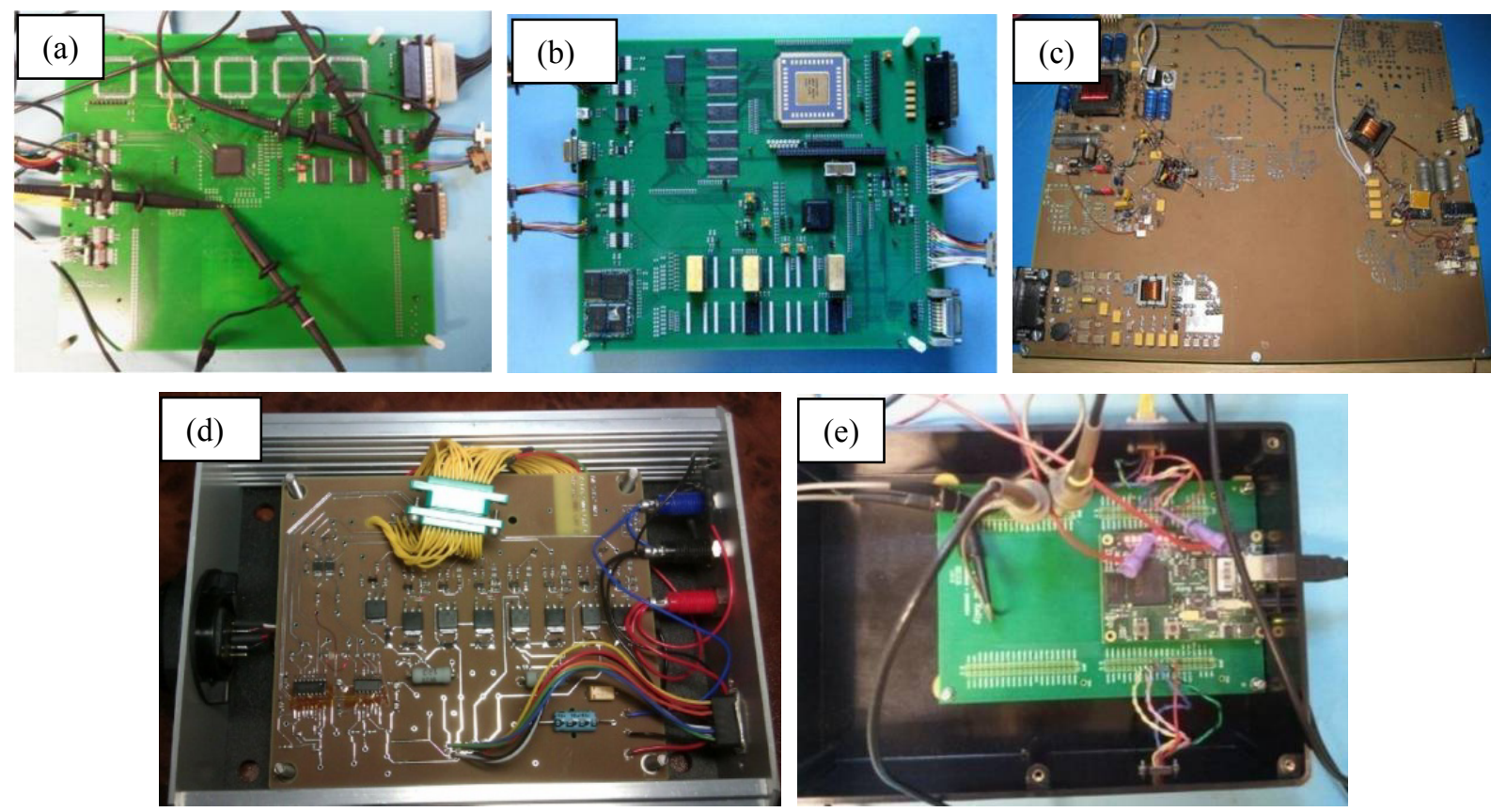

Figure 24 - CEB breadboard: (a) Compression, (b) Processor, (c) Power supply, (d) Auxiliary board (e) EGSE for High speed link

\subsection{Camera Front End Electronic (FEE)}

The FEE unit is quasi-identical for the three channels (apart the FSI sensor size). The FEE main components are:

- $\quad$ Control FPGA

- LVDS Driver/Receiver

- Analog to Digital Converter 
- $\quad$ An S(D)RAM memory

- $\quad$ ANALOG CONDITIONING

- $\quad$ The POWER I/F and Conditioning

Three dedicated breadboards (one for the APS interfaces definition, one for the ADC characterization and one for the FPGA design) were developed with the aim of grouping them in a complete representative FEE (Figure 25). The main outcome of this breadboard was to derive the flight model APS sensor requirements from an electrical point of view.

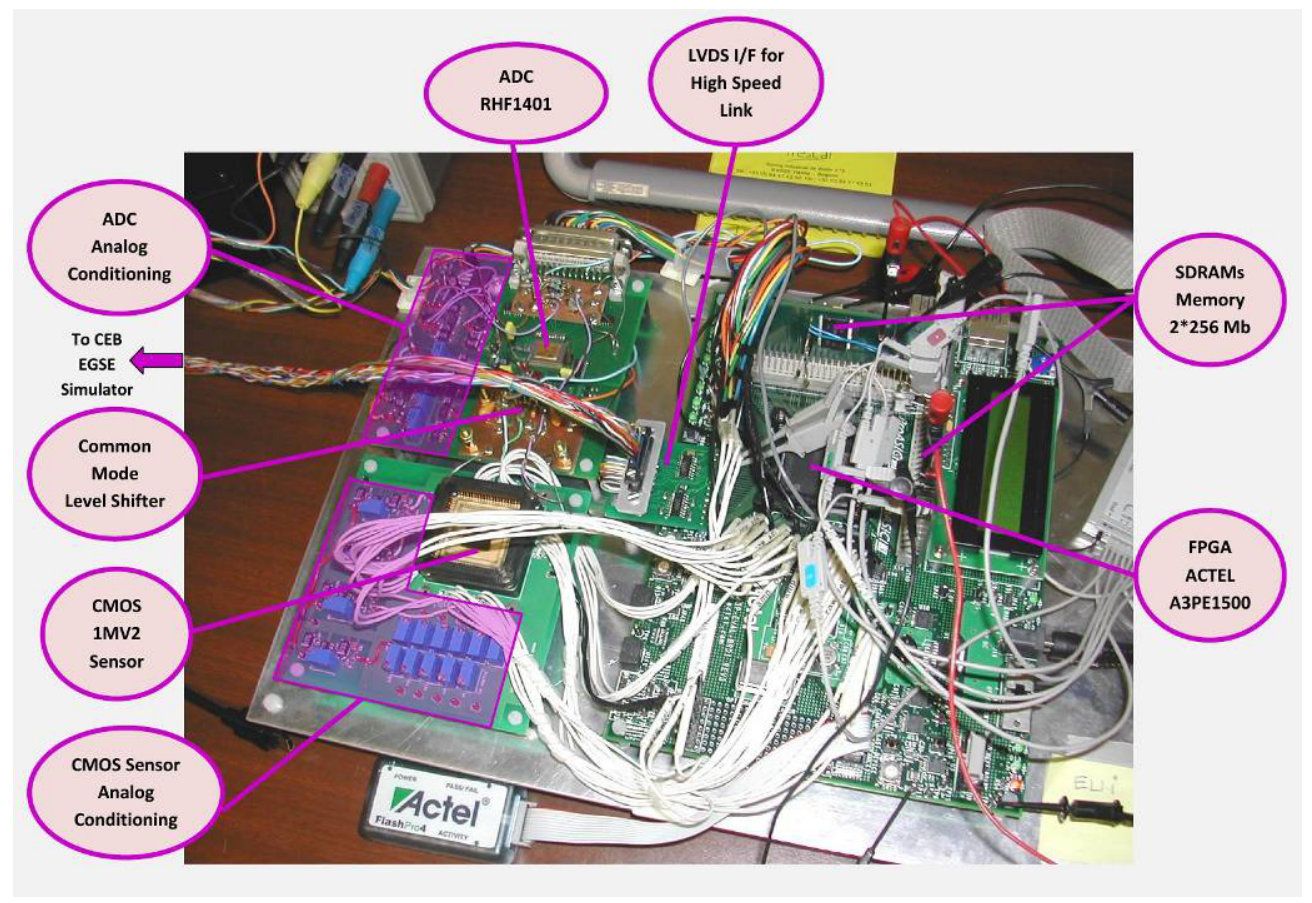

Figure 25 - The FEE breadboard, composed of three sub-units (APS interfaces, ADC and FPGA).

\subsection{Filter wheels mechanism}

The FSI filter wheel is a critical mechanism that needs to be qualified for 1 million operations, equivalent to 10 years of operations at 5 minutes cadence. HRI filter wheel number of operations is much less but its design is similar to the FSI one. A filter wheel breadboard has been manufactured and is under qualification. This breadboard is flight-like (mass, power consumption, envelope, and performances). It has been mechanical tested (Figure 26) and is currently in life time test (Figure 27) at $+50^{\circ} \mathrm{C}$ under vacuum for 3 months.

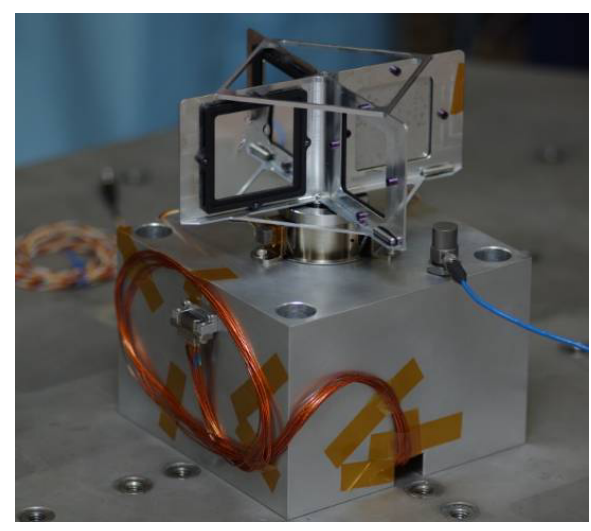

Figure 26 - FSI filter wheel during vibration tests.

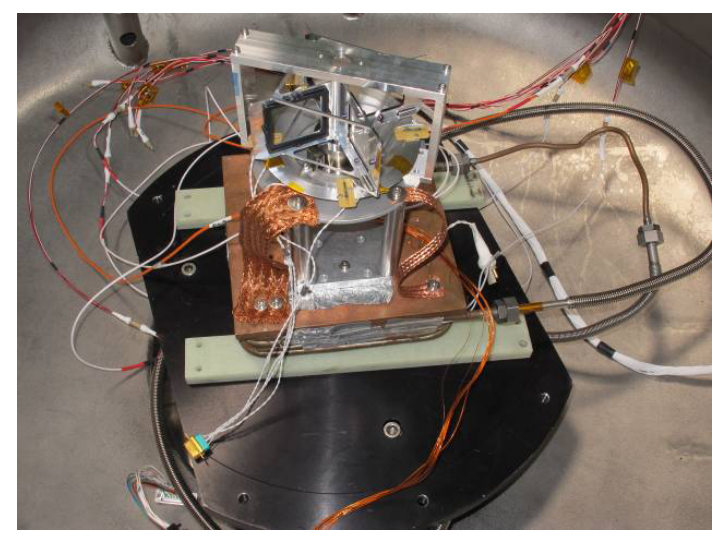

Figure 27 - FSI filter wheel breadboard ready for lifetime tests at $+50{ }^{\circ} \mathrm{C}$ under vacuum. 


\subsection{Internal doors mechanism}

A spacecraft mechanism is available on the heat shield to provide a first protection of the EUI instrument entrances. A set of three internal re-closable individual doors is intended to provide an additional contamination protection and protect the entrance filters during launch. The internal doors will also allow in-flight protection of the three EUI channels when EUI is not operated.

The current door design uses a sliding lid mechanism between the entrance baffle and the filter, sealing the end of the entrance baffle (similar to a gate valve). In the closed position, the door will be next to the entrance filter so as to minimize the volume between the door and the foil filter. This will reduce acoustic load to the foil filters during launch. The door on the baffle side will be a mirror, to maximize the heat rejection performance of the baffle. The same mechanism will be implemented in the two HRI and in the FSI channels.

A door breadboard has been manufactured to improve the design (motor selection, material choice...). This breadboard is flight representative in volume and material and was used to validate the concept (functional tests).

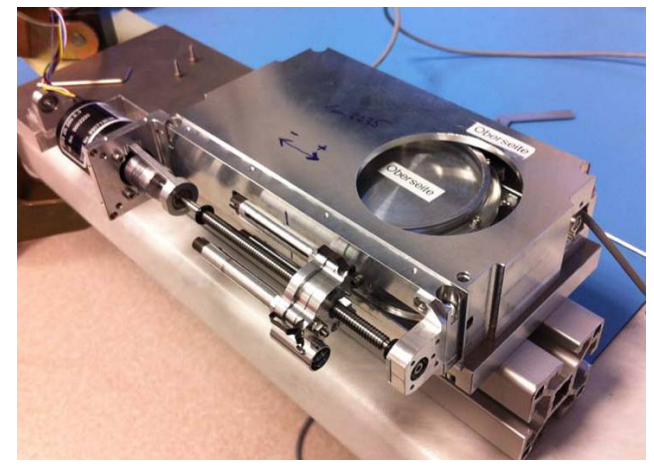

Figure 28 - The EUI internal door breadboard.

\subsection{Intensifier and high voltage unit}

The Lyman- $\alpha$ detector uses the same type of sensor (front side version of the EUV back-thinned detectors) and readout electronic circuits as the EUV channels. However, for better sensitivity at the Lyman- $\alpha$ wavelength, and for better suppression of solar continuum outside the filter pass band, the sensor will be mated with an image intensifier. The Lyman- $\alpha$ channel has indeed no metal filters like the EUV channels providing sufficient blocking of visible Sun light, but interference filters which need additional visible light blocking. This is accomplished by a blocking filter inside the MCP-intensifier, making it a solar-blind detector. The intensifier will use multichannel plates with a photocathode coating and the anode will be coupled to the sensor with a fiber optic taper, to reduce the image size to the sensor size.

Breadboards of the intensifier and of its associated high voltage unit (HVU) have been developed (Figure 29) and will be tested. The objectives are to validate the functionality of the design, verify the coupling of the intensifier on the APSsensor, determine the useful voltage range, verify the uniformity of response, determine the proper adaptation of the intensifier amplification with the dynamic range of the APS-sensor, characterize the dynamic range and resolution, and to perform radiometric measurements at $121.6 \mathrm{~nm}$.
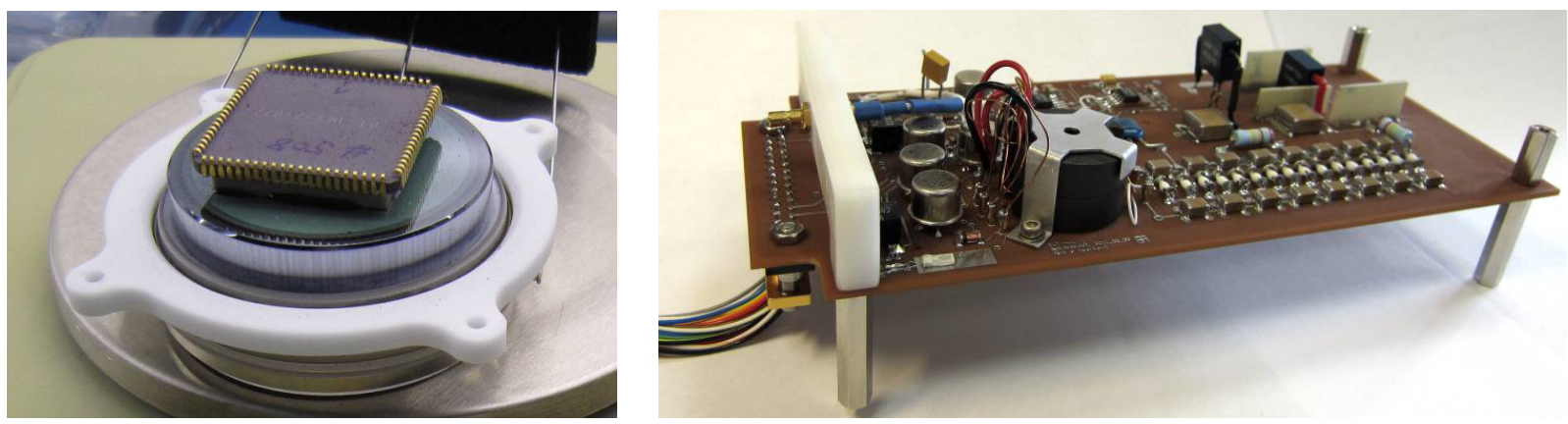

Figure 29 - Intensifier glued on a $1 \mathrm{k}$ x $1 \mathrm{k}$ APS prototype (left) with associated high voltage unit breadboard (right) 


\section{INSTRUMENT VERIFICATION AND QUALIFICATION}

\subsection{EUI instrument models}

The EUI model philosophy is summarized in Figure 30. It is organized around four models that will be manufactured and tested: one structural and thermal model (STM), one engineering model (EM), one qualification model (QM) and the flight model (FM). The EQM and some additional flight grade parts will serve as flight spare (FS).

\begin{tabular}{|c|c|c|}
\hline Model & Objectives & Representativity \\
\hline $\begin{array}{l}\text { Structural \& Thermal } \\
\text { Model (STM) }\end{array}$ & $\begin{array}{l}\text { - validation of thermal design and math models } \\
\text { - validation of structural design (primary structure) and } \\
\text { math models } \\
\text { - to derive subsystem environmental specifications }\end{array}$ & $\begin{array}{l}\text { - } \text { mounting interface } \\
\text { - mass properties } \\
\text { - structural properties } \\
\text { - thermal properties } \\
\text { - envelope and shape } \\
\text { - subsystems structural \& thermal dummies } \\
\text { - interconnecting harness }\end{array}$ \\
\hline Engineering Model (EM) & $\begin{array}{l}\text { - } \quad \text { validation of functional interfaces } \\
\text { - validation of TM/TC protocols } \\
\text { - } \quad \text { validation of operational procedures }\end{array}$ & $\begin{array}{l}\text { - electronics with flight layout with respect to } \\
\text { electrical parameters but populated with } \\
\text { lower-grade parts } \\
\text { - subsystems electrical dummies } \\
\text { - interconnecting harness }\end{array}$ \\
\hline Qualification Model (QM) & $\begin{array}{l}\text { - } \\
\text { - } \quad \text { endification of integration and alignment plans } \\
\text { - } \quad \text { verification of electrical performances, incl. EMC } \\
\text { - } \quad \text { validation of on-board software and functional } \\
\text { interfaces } \\
\text { - } \quad \text { instrument qualification }\end{array}$ & $\begin{array}{l}\text { - } \\
\text { - flight-like optics } \\
\text { redundional sub-equipments dummiec for } \\
\text { - full flight design \& flight standard with } \\
\text { respect to electrical parameters }\end{array}$ \\
\hline Flight Model (FM) & - flight use & - full flight design \& flight standard \\
\hline Flight Spare (FS) & - flight use (spare) & $\begin{array}{ll}\text { - } & \text { full flight design \& standard } \\
\text { - } & \text { refurbished EQM } \\
\text { - } & \text { electronic spare parts } \\
\end{array}$ \\
\hline
\end{tabular}

Figure 30 - EUI model philosophy

\subsection{EUI verification plan}

The Instrument verification will be performed incrementally at different verification levels:

- Equipment level. Every equipment, part or processes not having flight heritage will be subject to a dedicated qualification program.

- Subsystem level. As far as achievable, the main sub-assemblies will be pre-qualified, prior to integration into the instrument at unit level. The qualification of the primary structure through the STM program is an example of subsystem qualification.

- Instrument unit level, i.e. the fully assembled units. The verification stages at instrument level are covering the qualification of representative models, and the acceptance of the flight system. The test strategy that is deployed at instrument level is presented in test matrix Table 3 .

The verification of the instrument requirements will be achieved according to one or more of the four classical methods defined in ECSS-E-ST-10-02C, the selection of verification methods, levels, stages and associated models is a compromise between cost, schedule, performance and associated risks.

- Review of design (R). It consists of reviewing and approving a design report, a technical description and/or an engineering drawing for conformance to a specific requirement.

- Inspection (I). It is a verification method that determines conformance to requirements for constructional features, document and drawing conformance, workmanship and physical conditions without the use of special laboratory equipment, procedures or services. Inspections consist of controlling (mainly visually) the finished product against a predefined checklist.

- Analysis (A). It consists of performing theoretical or empirical evaluation by accepted techniques. The following engineering analyses are considered, when applicable: interface circuit analyses (in-rush current...), mechanical analyses (mass properties, stiffness, modal characteristics, quasi-static, sine, random accelerations, 
shock, bolt analysis, venting verification), thermal analysis, radiation analysis, part stress analysis / derating analysis, worst-case analysis, reliability analysis, safety analysis, failure propagation analysis (FMECA)...

- Test (T). It consists in a verification of the requirements by measuring product performance and function under various simulated environments. The verification process is implemented in subsequent verification stages all along the program life cycle. The verification stages are:

$\circ$ Qualification $(\mathrm{Q})$ : to demonstrate that the instrument will function within performance specifications under simulated conditions more severe than those expected from ground handling, launch and orbital operations. The purpose is to uncover deficiencies in design and method of manufacture and is not intended to exceed design safety margins or to introduce unrealistic modes of failure.

- Acceptance (A), to demonstrate that the instrument is acceptable for flight. It also serves as a quality control screen to detect deficiencies, and normally to provide the basis for delivery of the flight system to ESA.

A last stage of verification is performed once in orbit through a dedicated commissioning campaign.

Table 3 - Test matrix of EUI instrument models

\begin{tabular}{|c|c|c|c|c|c|}
\hline \multirow{2}{*}{ Test } & \multicolumn{5}{|c|}{ Model } \\
\hline & STM & EM & EQM & FM & FS \\
\hline Physical properties & $\mathbf{A}^{(1)}, \mathbf{T}$ & - & $\mathbf{A}^{(1)}, \mathbf{T}$ & $\mathbf{A}^{(1)}, T$ & - \\
\hline Functional \& performance ${ }^{(2)}$ & - & $T$ & $\mathbf{T}$ & $\mathbf{T}$ & $\mathbf{T}$ \\
\hline Static load & - & - & - & $\mathbf{A}$ & - \\
\hline Sinusoidal and random vibration & $\mathbf{T}_{\mathbf{Q}}$ & - & $\mathbf{T}_{\mathbf{Q}}$ & $\mathbf{T}_{\mathrm{A}}$ & - \\
\hline Acoustic & \multicolumn{5}{|c|}{ (tested at spacecraft level) } \\
\hline Shock & - & - & $\mathbf{T}$ & - & - \\
\hline Thermal balance & $T^{(3)}$ & - & $T^{(3)}$ & $T^{(3)}$ & - \\
\hline Thermal vacuum cycling & - & - & $\mathbf{T}_{\mathbf{Q}}^{(4)}$ & $T_{A}{ }^{(4)}$ & - \\
\hline Corona $\&$ arcing & & - & $\mathbf{T}$ & - & - \\
\hline${\mathrm{EMC} / \mathrm{ESD}^{(5)}}$ & - & $T^{(6)}$ & $\mathbf{T}^{(\mathbf{5})}$ & $\mathbf{T}$ & - \\
\hline Life & \multicolumn{5}{|c|}{ (tested at equipment level only, where required) } \\
\hline
\end{tabular}

$\mathbf{R}=$ Review of design $; \mathbf{A}=$ Analysis $\mathbf{I}=$ Inspection; $\mathbf{T}=$ Test $; \mathbf{T}_{\mathbf{Q}}=$ Test at qualification level; $\mathbf{T}_{\mathbf{A}}=$ Test at acceptance level

(1) $\mathrm{CoG}$ and Inertias verified by $\underline{\text { Analysis }}$

(2) Functional and health checks at ambient.

(3) With representative solar flux

(4) Thermal balance and cycling are combined in a single vacuum sequence

(5) Radiated Emission and susceptibility.

(6) Only conductive EMC tests

\subsection{Thermal validation}

The main constraint of the Solar Orbiter mission is its hard thermal environment, due to the close approach to the Sun when spacecraft is at perihelion.

In that context, the EUI instrument thermal constraints are the following:

- Limit the absorbed heat and evacuate the absorbed heat in the HRI entrance baffles to maintain their temperature and the entrance filters temperature below $120^{\circ} \mathrm{C}$. It is achieved by the entrance baffles rejecting most of the unwanted light and connected to one $\mathrm{S} / \mathrm{C}$ radiator.

- Evacuate the power dissipated by the detectors to maintain their temperature below $-40{ }^{\circ} \mathrm{C}$ in operational mode. It is achieved by connecting each detector to one cold radiator of the $\mathrm{S} / \mathrm{C}$.

- Evacuate the power dissipated by the detector front end electronics (FEE) to maintain their temperature below $60{ }^{\circ} \mathrm{C}$ in OP mode. It is achieved by connecting each FEE to one $\mathrm{S} / \mathrm{C}$ radiator.

- Ensure the optical bench thermo-elastic deformation remains acceptable between optical alignment at $20{ }^{\circ} \mathrm{C}$ and in flight OP modes. It is achieved by use of a honeycomb sandwich panel optical bench with low thermo-elastic expansion coefficient CFRP facesheets. 
- Maintain the temperature of internal sub-systems (mirror multilayers, mechanisms, filters...) in acceptable temperature range to limit ageing effects. This constraint is driven by the $\mathrm{S} / \mathrm{C}$ temperature $\left(+50{ }^{\circ} \mathrm{C}\right.$ in hot operational case). A radiative decoupling is however implemented with thermal blanket (MLI) and insulating mounts to limit the impact of the $\mathrm{S} / \mathrm{C}$ thermal variation, the bottom of the optical bench being not insulated to evacuate FEE dissipation by radiative exchange to the $\mathrm{S} / \mathrm{C}$ via the optical bench surface. Heaters are also foreseen on the mirrors and detectors for decontamination purpose.

- Maintain the detectors a few degrees hotter than their local environment. It is achieved by a dedicated camera design including a cold cup around the detector, which is colder than the detector itself

All these constraints will be verified at unit level as part of the thermal validation tests on the STM, EQM and FM models. The heat rejection of the EUI entrance baffle will however be first validated at sub-system level before tests at instrument level (i.e. as part of the thermal balance and thermal cycling of Table 3).

The thermal tests at instrument unit level will be performed in a dedicated test setup (Figure 31) at the Centre Spatial de Liège (CSL) facilities (Figure 32). The thermal verification is based on a similar heat flux obtained with a Xenon lamp for the entrance filter thermal tests (Figure 15) adapted to the instrument three entrance apertures. Two Xenon lamps, providing respectively $160 \mathrm{~mm}$ and $25 \mathrm{~mm}$ simulated collimated solar beam.

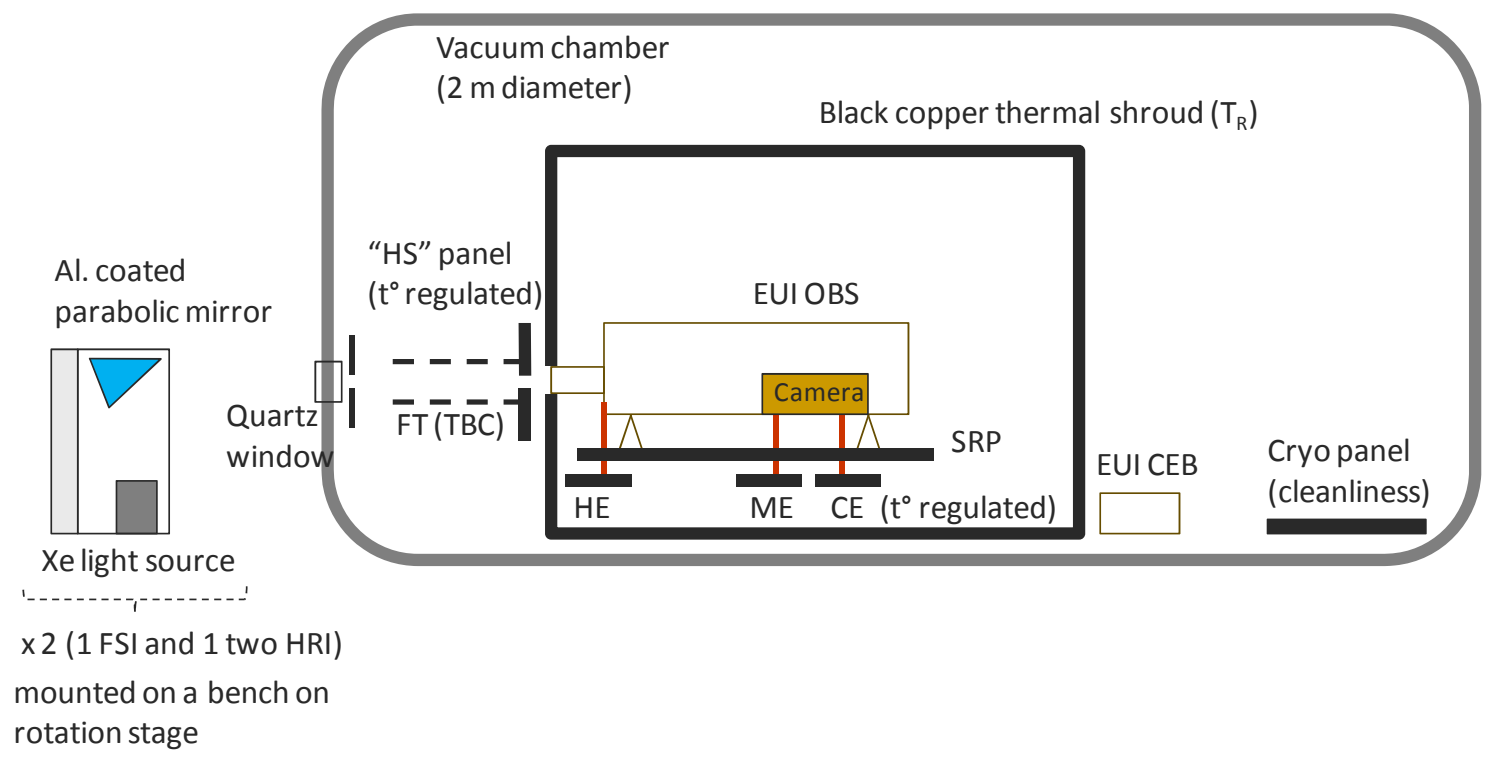

Figure 31 - Thermal balance test configuration, where two Xenon light sources are used to simulate the solar heat input.

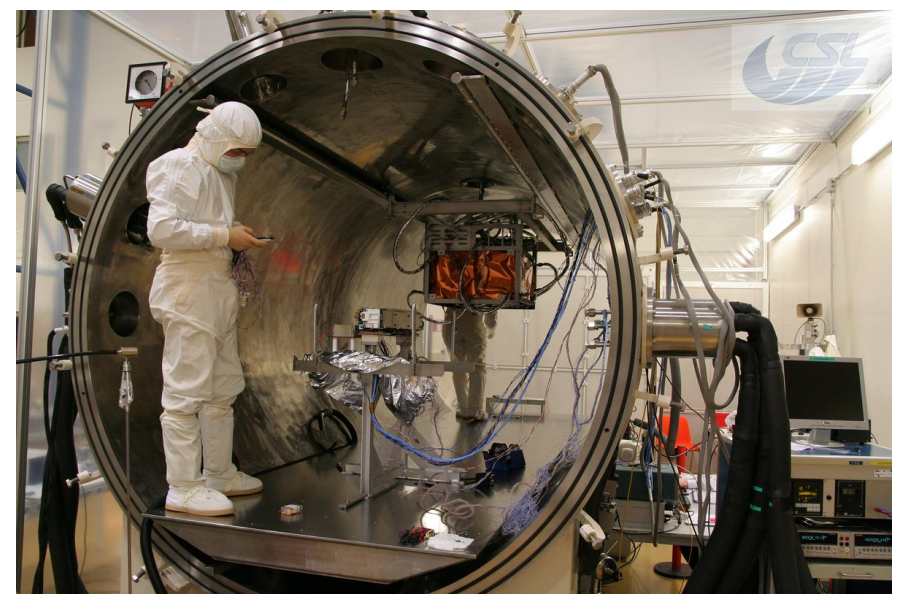

Figure 32 - The $2 \mathrm{~m}$ diameter vacuum chamber at the Centre Spatial de Liège will be equipped for the EUI instrument thermal balance and vacuum tests. Here the chamber is shown with the SWAP flight model instrument. 


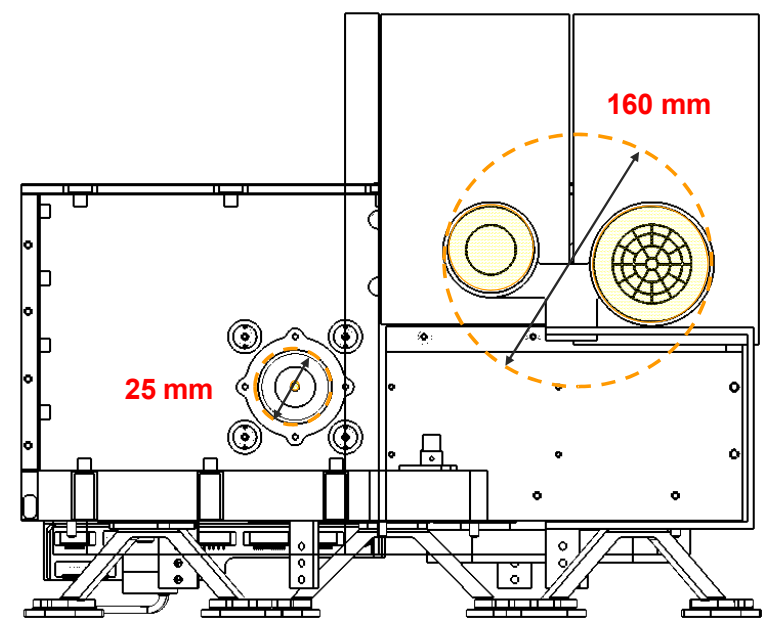

Figure 33 - Two beams will be used to simulate the solar heat input to the EUI entrance baffles.

\subsection{Flight model AIT}

Figure 34 shows the overview of the Assembly, Integration and Test flow of the EUI flight model instrument. It is composed of a dedicated flow for each of two OBS and CEB unit. The two flows are joined at the end for a set of end-toend integrated tests.

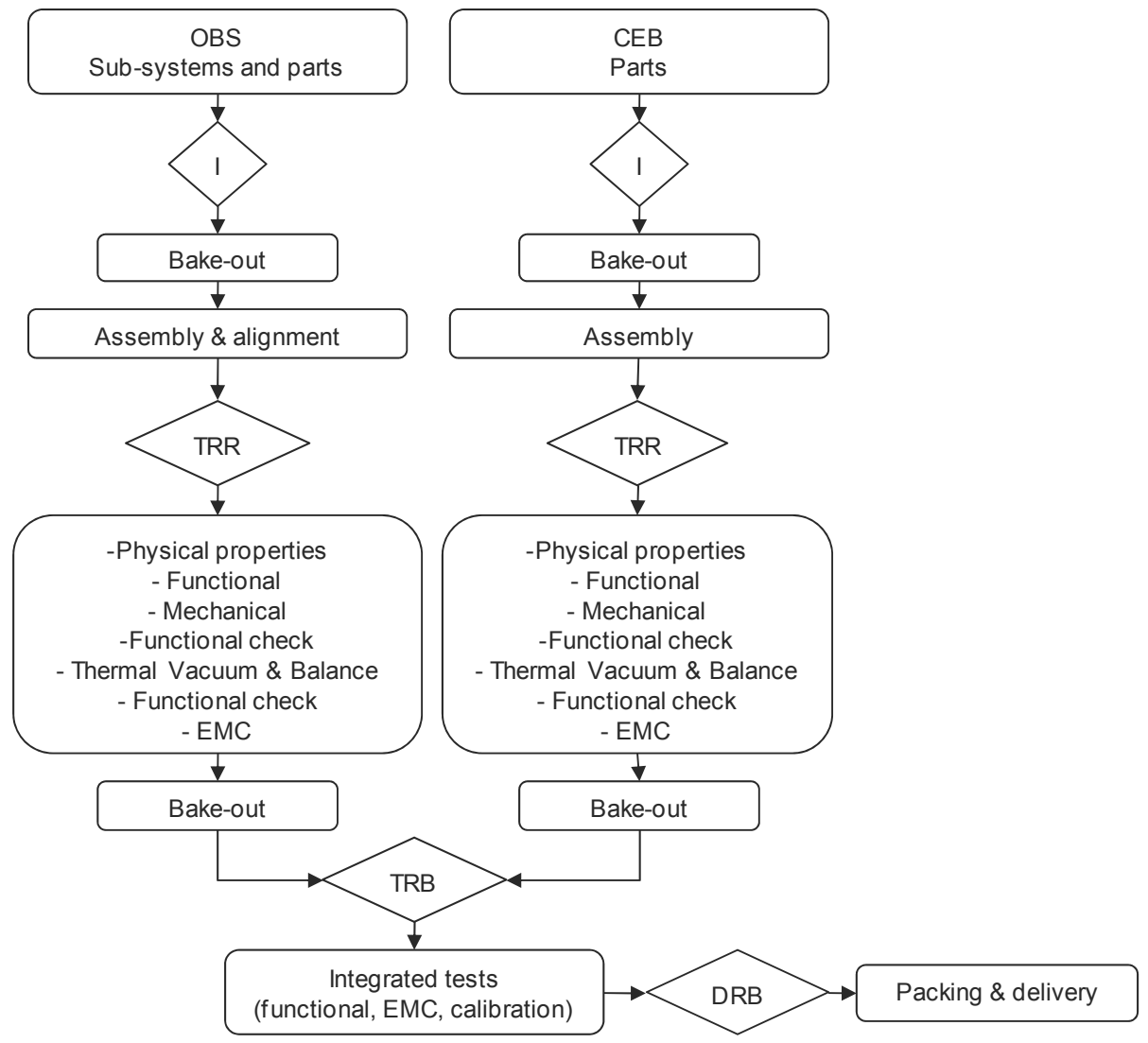

Figure 34 - AIT overview of the EUI flight model instrument. 


\section{CONCLUSIONS}

Solar Orbiter being an encounter mission, there will be a limited number of observation windows. It will also require synergies with other Solar Orbiter instruments (dedicated science per observation window) with optimization of onboard storage, telemetry and power.

Following a technological development process, the four identified key technologies of EUI have been tested and validated, as summarized in Table 4.

Table 4 - Key technologies validation

\begin{tabular}{|l|l|l|}
\hline \multicolumn{2}{|c|}{ Component } & \multicolumn{1}{c|}{ Validation } \\
\hline Entrance Baffles & HRIs & $\begin{array}{l}\text { Optical rejection matching theoretical performances } \\
\text { Manufacturing process (coating) validated on samples }\end{array}$ \\
\hline \multirow{2}{*}{ Filters } & FSI & $\begin{array}{c}\text { Transmission measured on representative filters Mechanical properties validated on filter } \\
\text { wheel breadboard }\end{array}$ \\
\cline { 2 - 3 } & HRI EUV & $\begin{array}{c}\text { Thermo-mechanical properties of representative filters as expected, including tests under } \\
13 \text { solar constants }\end{array}$ \\
\hline Mirrors coating & FSI / HRI & $\begin{array}{l}\text { Coating with improved reflection (single and dual band) as theoretically computed. } \\
\text { Temporal and thermal stability validated on samples. }\end{array}$ \\
\hline Detectors & FSI / HRI & $\begin{array}{c}\text { Performances tests (low noise, sensitivity...) of smaller array in standard packaging in } \\
\text { flight conditions, and after radiations }\end{array}$ \\
\hline
\end{tabular}

In addition, a set of breadboards have been manufactured and tested to confirm the preliminary design and start the instrument model procurement.

In the coming months, the STM model of the two instrument units (OBS and CEB) will be assembled and tested. In particular, the STM will be used to validate and correlate the thermal model but also to ensure the entrance baffle and its filter behave as expected, and to refine the thermal interfaces with the spacecraft.

\section{ACKNOWLEDGEMENTS}

The EUI instrument is developed in a collaboration which includes the Centre Spatial de Liège (Belgium), the Institut d'Astrophysique and Institut d'Optique (France), the UCL Mullard Space Science Laboratory (UK), the Max Planck Institute for Solar System Research (Germany), the Physikalisch-Meteorologisches Observatorium Davos (Switzerland), and the Royal Observatory of Belgium (Belgium)

The Belgian institutions are funded by Belgian Federal Science Policy Office (BELPSO); the French institutions by Centre National d'Etudes Spatiales (CNES), the UK institution by the UK Space Agency (UKSA); the German institution by Deutsche Zentrum für Luft- und Raumfahrt e.V. (DLR), and the Swiss institution by the Swiss Space Office (SSO).

\section{REFERENCES}

[1] Fleck B., Harrison R. A., Marsden R. G., Wimmer-Schweingruber R., "Summary of the Solar Orbiter payload working group activities, Telescopes and Instrumentation for Solar Astrophysics", Proc. SPIE 5171, 123-130 (2004).

[2] Marsden R.G., Marsch E. and the Solar Orbiter Science Definition Team, "Solar Orbiter Science Requirements Document", SCI-SH/2005/100/RGM Issue 1 Revision 2 (2005).

[3] Mc Coy D., and the Solar Orbiter assessment team, "Solar Orbiter Payload Definition Document", SCI$\mathrm{A} / 2004 / 175 / \mathrm{AO}$ Issue 5 Revision 0 (2006).

[4] Auchere F., Song X., Rouesnel F., Appourchaux T., Fourmon J.-J., Le Clec'h J.-C., Berthe M., Defise J.-M., Mazy, E., Rochus P., Mercier R., Ravet M.-F., "Innovative designs for the imaging suite on Solar Orbiter, Solar Physics and Space Weather Instrumentation”, Proc. SPIE 5901, 298-304 (2005). 
[5] Vial J.-C., "Solar Orbiter: A unique opportunity for investigating small-scale physical processes at work in the magnetic solar atmosphere, Advances in Space Research", Advances in Space Research 36, 1375-1386 (2005).

[6] Young P. R., and the EUS Science Working Group, "Science With The Extreme Ultraviolet Spectrometer For Solar Orbiter", Proc. of The Second Solar Orbiter Workshop 641 (2006).

[7] Rochus P., Halain J.P., Renotte E., Berghmans D., Zhukov A., Hochedez J.F., Appourchaux T., Auchère F., Harra L.K, Schühle U., Mercier R.., "The Extreme Ultraviolet Imager (EUI) on-board the Solar orbiter Mission”, 60th International Astronautical Congress (2009).

[8] Hochedez J.-F., Appourchaux T., Defise J.-M., Harra L. K., Schuehle U., Auchère F., Curdt W., Hancock B., Kretzschmar M., Lawrence G., Marsch E., Parenti S., Podladchikova E., Rochus P., Rodriguez L., Rouesnel F., Solanki S., Teriaca L., Van Driel L., Vial J.-C., Winter B., Zhukov A., "EUI, The Ultraviolet Imaging Telescopes of Solar Orbiter", The Second Solar Orbiter Workshop (2006).

[9] Halain J.-P., Rochus P., Appourchaux T., Berghmans D., Harra L., Schühle U., Auchère F., Zhukov A., Renotte E., Defise J.-M., Rossi L., Fleury-Frenette K., Jacques L., Hochedez J.-F., Ben Moussa A., "The technical challenges of the Solar-Orbiter EUI instrument", Proc. SPIE 7732, 26 (2010)

[10] Halain J.-P. , Berghmans D., Defise J.-M., Renotte E., Thibert T., Mazy E., Rochus P., Nicula B., De Groof A., Seaton D., Schühle U., "The First light of SWAP on-board PROBA2", Proc. SPIE 7732, 24 (2010)

[11] Auchère F., et al., "HECOR, a HElium CORonagraph aboard the Herschel sounding rocket", Proc. SPIE, 6689, 66890A-66890A-11 (2007)

[12] Schühle, U., Halain, J., Meining, S., Teriaca, L., "The Lyman-alpha telescope of the extreme ultraviolet imager on Solar Orbiter" in Solar Physics and Space Weather Instrumentation IV, edited by Silvano Fineschi, Judy Fennelly, Proc. SPIE 8148, 81480 (2011) 